\title{
Hidrogeología del Parque Nacional de los Picos de Europa, norte de España
}

\author{
Mónica Meléndez ${ }^{(1)}$, Daniel Ballesteros ${ }^{(2,3)}$ y Montserrat Jiménez-Sánchez ${ }^{(2)}$ \\ (1) Instituto Geológico y Minero de España, Calle Matemático Pedrayes, 25, 33005 Oviedo, España \\ m.melendez@igme.es \\ (2) Departamento de Geología, Universidad de Oviedo. Calle Jesús Arias de Velasco, s/n, 33005 Oviedo, España \\ (3) UMR 6266 IDEES, Université de Rouen Normandie-CNRS, 76821 Mont Saint-Aignan CEDEX, Francia
}

\begin{abstract}
RESUMEN
El Parque Nacional de los Picos de Europa, con una superficie total de $675 \mathrm{~km}^{2}$, está situado en el norte de España, en las provincias de Asturias, Cantabria y León. Su sustrato geológico está formado por materiales carbonatados en su parte central y septentrional, mientras que al Sur son mayoritarias las rocas silíceas. La compleja estructura geológica ocasionada por la superposición de dos orogenias, ha condicionado la presencia de series calcáreas de más de $2 \mathrm{~km}$ de potencia muy karstificadas en las que se han documentado el $14 \%$ de las simas más profundas del planeta. Desde el punto de vista de la planificación hidrológica, el Parque está integrado en las masas de agua subterráneas 016.214 Picos de Europa-Panes y 016.218 Alto DevaAlto Cares. Las formaciones geológicas presentes en el Parque Nacional de los Picos de Europa, considerando las características de permeabilidad de los materiales, se pueden clasificar como acuíferos o acuitardos. El acuífero principal es de naturaleza kárstica e incluye una potente serie de calizas carboníferas de orientación O-E. La presencia de formaciones siliciclásticas que se comportan como acuitardos condiciona la compartimentación del acuífero en "cuerpos de agua subterránea menores" (en adelante SGWB, acrónimo de la terminología inglesa, "smaller groundwater bodies") de dimensiones entre 2 y $108 \mathrm{~km}^{3}$ de volumen. La cota del nivel piezométrico en los distintos SGWBs varía entre 120 y 1.200 m s.n.m., descendiendo de forma escalonada hacia el norte. Cabe destacar que en los Macizos Central y Oriental la piezometría es más uniforme y se ubica entre 300 y 500 m s.n.m. Al norte del límite del Parque, los principales puntos de descarga del acuífero carbonatado se sitúan a cotas entre 120 y 150 m s.n.m. La recarga del acuífero, preferentemente por infiltración directa, presenta una clara influencia nival. Las surgencias kársticas descargan conjuntamente las aguas procedentes de la escorrentía superficial infiltradas en el acuífero y las subterráneas que proceden de distintos SGWBs. La presencia de unidades siliciclásticas, con direcciones generales O-E que funcionan como barreras de permeabilidad ha condicionado que la circulación del flujo subterráneo se dirija predominantemente en sentido Norte, y por tanto, ha propiciado que las principales surgencias se ubiquen en la parte septentrional del Parque.
\end{abstract}

Palabras claves: Picos de Europa, agua subterránea, hidrodinámica, hidrogeología, karst.

\section{Hydrogeology of the Picos de Europa National Park (northern of Spain)}

\begin{abstract}
The Picos de Europa National Park, with a total area of $675 \mathrm{~km}^{2}$, is located in the North of Spain, in the provinces of Asturias, Cantabria and León. Carbonated materials predominate in the North and central areas of the Park, whilst to the South rocks are mainly siliceous. The complex geological structure caused by the superposition of two orogenies has conditioned the presence of a very karstified limestone series more than $2 \mathrm{~km}$ thick. The karst development is really important and it is well known that the Picos de Europa high mountains contain $14 \%$ of the deepest discovered caves in the world. As far as hydrological planning is concerned, the park is integrated into the 016.214 Picos de Europa-Panes and 016.218 Alto Deva-Alto Cares groundwater bodies. The geological formations present in the Picos de Europa National Park, considering the permeability characteristics of the materials, can be classified as either aquifers or aquitards. The main aquifer is karst in nature and includes a powerful series of carboniferous limestones with an O-E orientation. The presence of siliciclastic formations, which behave like aquitards, determine the compartmentalization of the aquifer into "smaller underground water bodies" (hereinafter SGWB, the acronym for the English terminology, "smaller groundwater bodies") with dimensions of between 2 and $108 \mathrm{~km}^{3}$ in volume. The piezometric level in the dif-
\end{abstract}


ferent SGWBs varies between 120 and 1,200 $\mathrm{m}$ a.s.I., descending in a staggered way to the North. It should be noted that in the Central and Eastern Massifs the piezometry is more uniform and is located between 300 and $500 \mathrm{~m}$ a.s.l. To the northern of the limit of the park, the main points of discharge of the carbonated aquifer are located between 120 and $150 \mathrm{~m}$ a.s.l. The aquifer recharge, preferably by direct infiltration, has a clear snow influence. The karst springs jointly discharge the water from the surface runoff infiltrated into the aquifer and the groundwater that comes from the different SGWBs. The presence of siliciclastic units, with general $W$-E directions that function as permeability barriers has caused the circulation of the underground flow to be directed predominantly northwards, and therefore, has led to the main springs being located in the northern part of the park.

Keywords: Picos de Europa, groundwater, hydrodynamic, hydrogeology, karst.

\section{ABRIDGED ENGLISH VERSION}

\section{Introduction}

The Picos de Europa National Park, with total area of $675 \mathrm{~km}^{2}$, is located in the North of Spain, between the Autonomous Communities of Castilla y León, Cantabria and Principality of Asturias (Fig. 1). Its high environmental and ecological value has led it to be declared as a National Park (1918 and 1996) and a Biosphere Reserve (2003); within the park there are also six Sites of Community Interest, five Areas of Special Protection for Birds and four karst caves declared as Natural Monuments. Geomorphological processes related to surface water and groundwater are very significant in landscape modelling and karst development. Groundwater accounts for approximately $80-90 \%$ of the water resources of the area, which have been estimated at between 520 and $720 \mathrm{hm}^{3} /$ year; the water resources are used for human and livestock supply, with livestock and tourism being the main economic activities of the region.

It is worth mentioning that, from a geological heritage point of view, the "Picos de Europa" karst is important at both a national and international scale since the Geological and Mining Institute of Spain (IGME) declared it to be Global Geosite in 2007. In addition, it is included in the "Geological Contexts of Spain with global relevance" as reflected in Law 42/2007, of the Natural Heritage and Biodiversity of Spain and includes four Geological Interest Sites.

Speleological expeditions are particularly relevant not only for cave surveying but also for karst water connections that have been researched through the use of tracers. At the end of the 1990s, groundwater investigations provided the park with a basic hydrogeological infrastructure, which together with other complementary studies, created the first estimates of its reserves and resources. Initially, it was included in the "Hydrogeological System number 3. Cantabria-Astur Mountain Limestone. Unit 3A. Picos de Europa" and when the Hydrogeological Units were defined, it became part of the "UH. 01.17 Picos de Europa-Panes". With regard to the delimitation of "groundwater bodies (GWB)", following the criteria established in the Water Framework Directive (2000/60 / CE), the National Park includes the groundwater bodies 016.214 Picos de Europa-Panes and the 016.218 Alto Deva-Alto Cares.

The climate of the park presents average monthly temperatures of between -3 and $20^{\circ} \mathrm{C}$, which favour the development of warm summers and the formation of a snow mantle during 6 to 9 months of the year. The daily thermal variations are from 5 to $15^{\circ} \mathrm{C}$, even during the strong frosts of winter, favouring the rapid melting of the snow mantle. The climate has a remarkable oceanic influence. The annual precipitation ranges from 1,000 to $1,800 \mathrm{~mm}$, although in some eastern valleys, Mediterranean microclimates occur with $670-700 \mathrm{~mm}$ of annual precipitation, although without a dry season.

The river network is formed by three main rivers: the Sella, the Deva and its tributary, the Cares, together with small tributaries, with the main flow component being to the North. These rivers are located at levels between 100 and 1,000 m s.n.m., highlighting the existence of river canyons with depths of up to 2,000 m, such as the Desfiladero de los Beyos and the Garganta del Cares (Fig. 2)

From the geological point of view, the National Park is part of two geological units of the Cantabrian Zone: the Bodón-Ponga and the Pisuerga-Carrión (Fig. 3). The predominant materials are Carboniferous limestone in the North and central zones of the park, whilst in the South, the siliceous Estefanian rocks predominate. The complex geological structure of the Picos de Europa, caused by the superposition of two orogenies, explains the presence of a limestone series more than $2 \mathrm{~km}$ thick. The strong incision of the fluvial network together with the predominance of a humid climate during part of its Cenozoic evolution, have caused a large karst development. In this karst, speleological groups have been documented as making up $14 \%$ of the deepest shafts of the planet. 


\section{Results and discussion}

The geological formations present in the Picos de Europa National Park, as far as the permeability characteristics of the materials is concerned, can be classified as either aquifers or aquitards. The Lower Paleozoic Aquitard, the Karstic Carboniferous Aquifer, the Aquitard of the Upper Carboniferous sequence, the Syorogenic Carboniferous Aquitard, the Detritic Carboniferous Aquitard and Aquifer and the Quaternary Aquitard (Figs. 4 and 5).

The main aquifer is karst in nature and includes a powerful series of carboniferous limestones, with a WE orientation, which crops in most of the park surface (Fig. 7). On the other hand, the siliciclastic formations behave like aquitards due their low hydraulic conductivity. In addition, the quaternary surface formations may constitute small surface aquifers of little interest or behave as aquitards depending on their lithological characteristics. The presence of aquitards determines the compartmentalization of the aquifer into "smaller underground water bodies" (SGWB) with dimensions of between 2 and $108 \mathrm{~km}^{3}$ in volume, which include reduced saturated zones and unsaturated zones up to 2,300 $\mathrm{m}$ thickness (Fig. 7).

The compartmentalization of the karst aquifer is due to the presence of low or very low permeability formations (aquitards) that act as permeability barriers, conditioning the existence of partially independent sectors whose volumes are variable (values have been described for the saturated zone of between 0.01 and 60 $\mathrm{km}^{3}$, Ballesteros et al., 2013, 2015c). The basal impermeable edge is made up of the materials of the PisuergaCarrión Unit, which are arranged, by mechanical contact, under the karst aquifer, the contact between them coinciding with the level of take-off of the variscal riding. In the South, this limit is inclined between 30 and $60^{\circ} \mathrm{N}$, whilst in the North, its inclination varies between $60^{\circ} \mathrm{N}$, verticalizing at depth (Figs. 6 and 7).

The recharge of the aquifer is carried out, preferably, by direct infiltration through the exokarst. It is estimated an infiltration coefficient of between 65 and 100\% from the effective rainfall for Picos de Europa. The most significant fluvial courses are Cares, Casaño, Dobra, Duje, Hunhumia, Pomperi and La Beyera rivers, with influent behaviour sectors in the Cares, Duje and Pomperi rivers. The discharge of the karst aquifer takes place through springs that drain certain areas or compartments of the aquifer (SGWB). It should be noted, therefore, that through the same spring (or several associated springs) surface water and groundwater of an area can comprise one or several compartments of the karstic aquifer that are drained together. In general, it has been observed that these drainage areas have surfaces ranging from 1 to $100 \mathrm{~km}^{2}$ and show an elongated arrangement following the E-W direction (Fig. 7)

The groundwater flow takes place mainly through well-developed karst conduits and is conditioned by the presence of siliciclastic units that work as permeability barriers. The inclination of these units towards the North causes the regional underground flow, also going to the North, a relevant feature to explain the location of the main karst springs in the northern Picos de Europa. However, at the local level, tracer tests show that groundwater can flow following different directions, to the north, northwest, northeast, and east (Table 1). These directions are generally parallel to the bedding thrusts and limits of the aquifer.

The discharge of the aquifer takes place through more than 20 springs, mostly located in the river valleys, near the riverbeds. These springs constitute the main contributions to the Casaño, La Beyera, Pomperi and Hunhumia rivers. The average drained flows vary from more than $250 \mathrm{l} / \mathrm{s}$ to less than $50-100 \mathrm{l} / \mathrm{s}$. During high water conditions, the flow can increase by one or two orders, conditioned by significant recharge variations and the high transmissivity of the aquifer. The flows drained through the upwellings are closely related to the extension of the recharge area of each of them, estimating an annual discharge of $3 \pm 1 \mathrm{hm} 3$ per $\mathrm{km} 2$ of drained surface (Fig. 8). The location of the main springs is conditioned by the presence of low permeability materials and regional scale faults, probably alpine, which cut previous structures. On the other hand, in some cases, there may be some hydrogeological connection between the water tables located at different heights, so the water tables with higher altitudes would recharge the levels located at lower elevations.

The altitude of the springs (Table 2), as well as the altitude of discharge points to the channels, has allowed us to infer that the piezometric surface is located at dimensions of between 145 and 1,425 $\mathrm{m}$ a.s.l. descending, in general, in a staggered way to the North, with differences between the piezometric levels of the order of 100 to $300 \mathrm{~m}$ and a maximum value of $600 \mathrm{~m}$. All this is compatible with the compartmentalization of the aquifer due to the influence of the tectonics mentioned above.

From the hydrochemical point of view, the karst waters of Picos de Europa are calcium bicarbonate (Fig. 9), have temperatures between 2.5 and $11^{\circ} \mathrm{C}, \mathrm{pH}$ values between 7.9 to 8.9 and the electric conductivity varies between 120 and $250 \mathrm{~S} / \mathrm{cm}$, decreasing with altitude. It is worth noting the presence of thermal waters in the Park; in the eastern limit, several thermal springs are located in the Deva River, one of which with temperatures reaching more than $60^{\circ} \mathrm{C}$.

Currently, the main uses of the groundwater of Picos de Europa National Park are the human and livestock supply, followed by hydraulic power generation and, in to a lesser extent, irrigation. Amongst the uses of groundwater in the past, its use for supplying traditional mills until the 20th century should be highlighted (examples of Caín and Sajambre in León, and Tresviso in Cantabria). 


\section{Introducción}

El Parque Nacional de los Picos de Europa (PNPE), en su mayor parte de naturaleza carbonatada, está situado en la cordillera Cantábrica, en el norte de España. Con una extensión de 67.500 ha distribuidas entre Cantabria, Asturias y León, presenta un elevado valor ambiental y ecológico (Barquín et al., 2015). En 1918, la zona occidental fue declarada Parque Nacional de la Montaña de Covadonga, siendo el primer espacio protegido del país, con una extensión de 16.925 ha. En 1995, ampliada su extensión a los sectores central y oriental, pasó a denominarse Parque Nacional de los Picos de Europa. Además, es Reserva de la Biosfera desde 2003 y cuenta con la presencia de 6 Lugares de Interés Comunitario (LIC) y 5 Zonas de Especial Protección para las Aves (ZEPA). Especialmente relevantes son también, las cuatro cavidades kársticas del Parque que han sido declaradas Monumento Natural por el Principado de Asturias por su excepcional interés geológico y geomorfológico.

Desde el punto de vista del patrimonio geológico, el karst de los Picos de Europa es un referente a escala nacional e internacional ya que fue declarado Global Geosite en 2007 por el Instituto Geológico y Minero de España (Durán Valsero y Robledo Ardila, 2009). Por otro lado, los Picos de Europa se incluyen dentro de los denominados "Sistemas kársticos en carbonatos y evaporitas de la Península lbérica y Baleares", considerados "Contextos geológicos de España con relevancia mundial" por la Ley 42/2007, del Patrimonio Natural y de la Biodiversidad de España (Carcavilla et al., 2009). Además, esta misma Ley incluye "los Sistemas kársticos en carbonatos y evaporitas" de España dentro de la relación de "unidades geológicas más representativas de la Geodiversidad del territorio español", reconociendo así la relevancia que tiene el karst de España a nivel mundial. Por último, cabe mencionar que cinco de los Lugares de Interés Geológico (LIG) ubicados en el Parque Nacional están relacionados con la hidrogeología, según consta en el Inventario Español de Lugares de Interés Geológico (IELIG). Dichos LIG, según figura en la base de datos de ELIG del IGME (http://info.igme.es/ielig/), son:

CA008 (Geosite SK004). Karst del Macizo de Cornión (Picos de Europa); CA008b (Geosite SK004b). Karst del Campo de los Senderos (Picos de Europa); CA107. Desfiladero de los Beyos; CA108. Garganta del Cares; y CA110. Poljé de Liordes.

Los importantes recursos hídricos del Parque Nacional, unidos a su interés ecológico y ambiental, así como al aprovechamiento de sus aguas subterrá- neas, han propiciado el desarrollo de estudios hidrogeológicos desde los años 60 , coincidiendo con el inicio de la exploración espeleológica de los Picos de Europa. Así, la investigación asociada a las actividades espeleológicas, junto con la elaboración de topografías de las cavidades y la localización de sus entradas, han permitido obtener numerosos datos sobre el funcionamiento hidrogeológico del karst. Entre los primeros estudios realizados, el grupo espeleológico Oxford University Cave Club (UK) estableció mediante ensayos de trazador algunas trayectorias y analizó el quimismo de las aguas subterráneas (Austin y Wilcock, 1961; Judson, 1963); posteriormente otros grupos nacionales y extranjeros siguieron en esta misma línea. En total, hasta 1990 los grupos espeleológicos llevaron a cabo 15 ensayos de trazadores y numerosos análisis químicos (Firman, 1979; Fabre y Fabriol, 1984; Borreguero, 1986; Collignon, 1986; Lloyd, 1990). En la siguiente década comienza de forma sistemática la investigación hidrogeológica en España en el marco del Plan Nacional de la Minería. En su ejecución, el IGME desarrolló el Programa "Mapa Hidrogeológico Nacional" que constó de dos fases: la primera dio lugar a los Mapas de Lluvia Útil y de Reconocimiento Hidrogeológico (escala $1: 1.000 .000)$ y al Mapa de Síntesis de Sistemas Acuíferos (escala 1:1.500.000) mientras que, durante la segunda, dentro del denominado Plan de Investigación de Aguas Subterráneas (PIAS), se realizó la primera investigación hidrogeológica en la Cuenca Norte (IGME, 1982; IGME, 1984). A nivel general, los principales resultados alcanzados se podrían resumir en: la creación de una infraestructura general hidrogeológica elaborando mapas hidrogeológicos a distintas escalas; un inventario de puntos de aguas recopilado en una base de datos; la caracterización hidroquímica del agua subterránea; la definición de los principales acuíferos y la cuantificación aproximada de sus recursos y reservas y, por último, la evaluación de las demandas actuales junto con la estimación de su evolución futura. Entre los trabajos realizados, figura la realización del primer inventario de puntos de agua en los Picos de Europa y la toma de datos de caudales en cursos de agua y manantiales. Cabe destacar, los resultados sobre la velocidad de flujo subterráneo y el análisis de la descarga de algunos manantiales (Virgós Soriano et al., 1980; Virgós Soriano, 1981). Además, se procedió a la caracterización hidroquímica del agua subterránea. Aunando todos los datos, el IGME realizó una primera estimación sobre los recursos hídricos de los Picos de Europa y estableció una primera hipótesis sobre la distribución de los potenciales hidráulicos del acuífero (IGME, 1982; IGME, 1984). 
La investigación llevada a cabo en el desarrollo del PIAS constituye la base hidrogeológica para establecer la ordenación y gestión de las aguas subterráneas en las siguientes décadas. La unidad de gestión definida se denominó "Sistema Hidrogeológico". El área de estudio se encuentra dentro del "Sistema Hidrogeológico $n^{\circ} 3$. Caliza de Montaña CántabroAstur. Unidad 3A. Picos de Europa" (IGME, 1982, IGME, 1984). Posteriormente, para una mejor gestión de los recursos hídricos, se definieron las Unidades Hidrogeológicas (UH) (DGOH, 1990), quedando el PNPE incluido en la UH. 01.17 Picos de Europa-Panes. Y por último, la transposición a la legislación española de la Directiva Marco del Agua (Directiva 2000/60/CE del Parlamento Europeo y del Consejo, de 23 de octubre de 2000, por la que se establece un marco comunitario de actuación en el ámbito de la política de aguas) conllevó la delimitación de masas de agua subterránea (MASb) como unidades básicas de gestión, siendo el Parque integrante de las MASb 016.214 Picos de Europa-Panes y 016.218 Alto DevaAlto Cares.

En respuesta a las necesidades crecientes del Parque Nacional en cuestiones relacionadas con estos usos del agua subterránea, con posterioridad a los anteriormente indicados, se realizaron otros trabajos de investigación aplicada; así, en López Geta et al. (1997) se estimaron los recursos hídricos de los

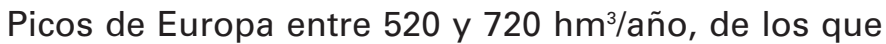
las aguas subterráneas representan aproximadamente el $80-90 \%$. Otro estudio aplicado es el de Meléndez-Asensio et al., 1998, quienes obtuvieron un modelo de funcionamiento hidrogeológico de la zona de los Lagos de Covadonga-Comeya en el Macizo Occidental del Parque. Ya en el siglo XXI, en la última década, se continuaron los estudios hidrogeológicos de los Picos de Europa por parte del Instituto Geológico y Minero de España y de la Universidad de Oviedo, proporcionando nuevos datos acerca de la geometría de los acuífero kársticos, su funcionamiento y la composición química de sus aguas (Ballesteros et al., 2013, 2014, 2015c; Meléndez et al., 2015). Estos estudios se enmarcan en el proyecto "Investigación hidrogeológica en las masas de aguas subterráneas 016.214 Picos de Europa-Panes y 016.218 Alto DevaAlto Cares" (IGME 2256/2013), financiado por el Instituto Geológico y Minero de España, y en el proyecto "Caracterización geomorfológica y geocronológica de cavidades kársticas en el Parque Nacional de Picos de Europa" (MAGRAMA 580/12), financiado por el Organismo Autónomo de Parques Nacionales (OAPN, Ministerio de Agricultura, Alimentación y Medio Ambiente de España). Además, con la colaboración de la Universidad de Oviedo, el IGME, conjun- tamente con el OAPN, editó la "Guía Geológica del Parque Nacional de los Picos de Europa" (Adrados et al. 2010), en la que a modo divulgativo, se recogen los principales aspectos geológicos e hidrogeológicos del Parque. Los nuevos datos, unidos al conocimiento previo, permiten obtener una nueva visión de la hidrogeología del Parque Nacional de los Picos de Europa.

En este amplio contexto se sitúa el objetivo de este trabajo, que, partiendo de la revisión y síntesis de los estudios hidrogeológicos realizados en el Parque Nacional, pretende: 1) actualizar el estado del conocimiento hidrogeológico en esta área protegida y 2) redefinir y proponer un nuevo modelo conceptual de funcionamiento hidrogeológico del Parque, basado, fundamentalmente, en los logros científicos más recientes.

\section{Situación}

El Parque Nacional de los Picos de Europa se localiza en el norte de España, abarcando territorios pertenecientes a las comunidades autónomas de Castilla y León, Cantabria y Principado de Asturias (Figura 1). La superficie actual del Parque (después de la ampliación aprobada el Acuerdo del Consejo de Ministros de 4 de Febrero del 2015) asciende a 67.455 ha y se extiende sobre once Términos Municipales, de los cuales tres (Oseja de Sajambre, Posada de Valdeón y Tresviso) están íntegramente dentro de los límites del Parque. La población estable dentro del Parque es del orden de 1.100 personas (OAPN, 2016).

\section{Marcos geográfico y geológico}

Los Picos de Europa constituyen un relieve agreste de media y alta montaña, de hasta $2.648 \mathrm{~m}$ de altitud, ubicado en la vertiente norte de la Cordillera Cantábrica (Figura 1). El clima del Parque Nacional se corresponde con los tipos Dfb y Dfc de la clasificación de Köppen, con temperaturas medias mensuales de entre -3 y $20^{\circ} \mathrm{C}$, que favorecen el desarrollo de veranos frescos o fríos y la formación de un manto nival de entre 6 y 9 meses de duración al año. Las variaciones térmicas diarias se estiman en $5-15^{\circ} \mathrm{C}$, incluso durante las fuertes heladas del invierno, favoreciendo rápidas fusiones del manto nival. El clima presenta una notable influencia oceánica con 1.000-1.800 mm de precipitación anual, si bien, en algunos valles orientales se dan microclimas mediterráneos con $670-700 \mathrm{~mm}$ de precipitación anual, aunque sin estación seca. 


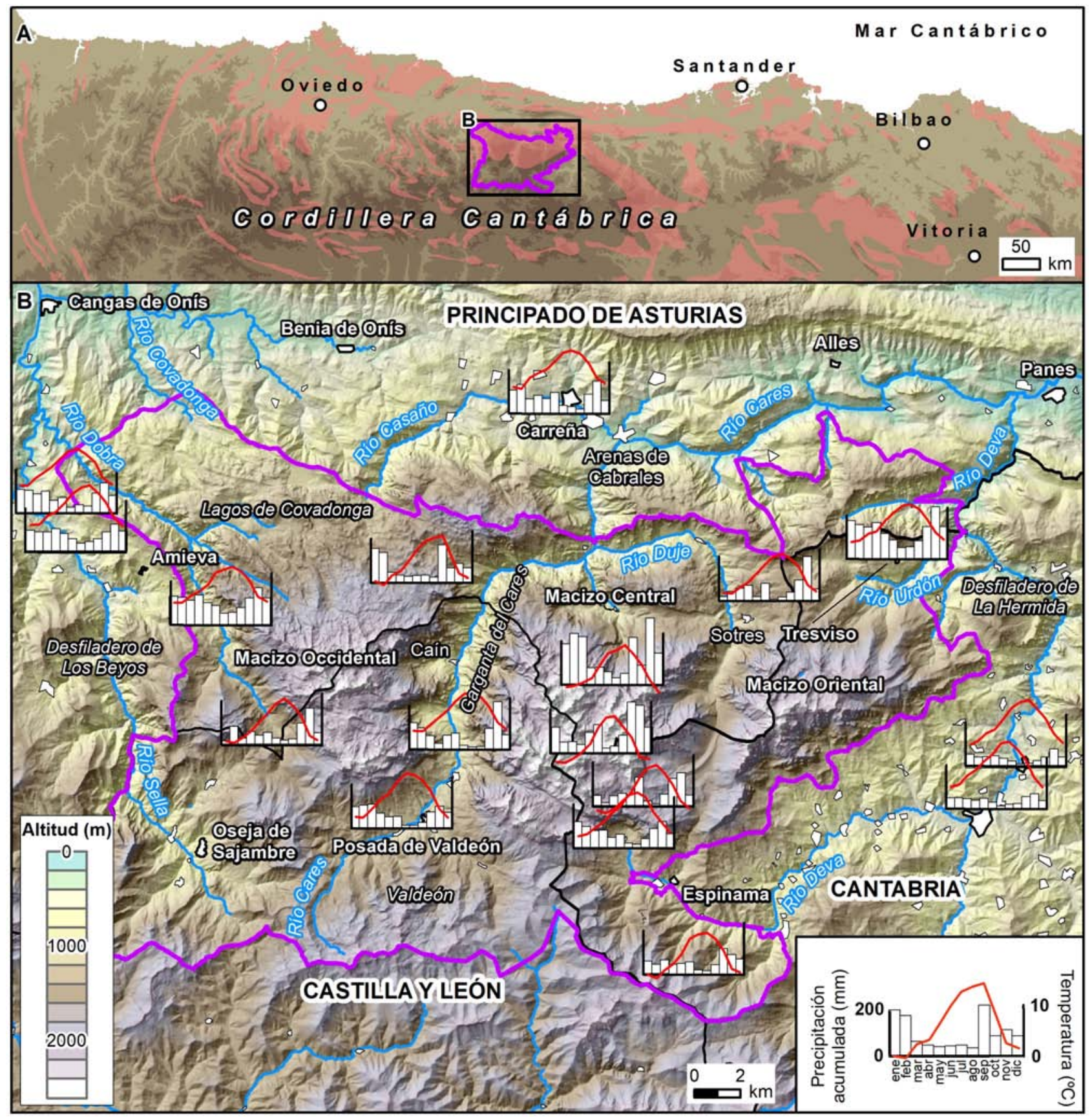

Figura 1. A Localización del Parque Nacional de los Picos de Europa en el ámbito de las áreas kársticas de la Cordillera Cantábrica (las tonalidades rosadas se corresponden con las áreas kársticas). B Relieve, red fluvial y climogramas del Parque Nacional confeccionados a partir de datos de 8 estaciones meteorológicas de la Red de Seguimiento del Cambio Global en Parques Nacionales (MAGRAMA, 2014) y de 9 estaciones de la Agencia Española de Meteorología (AEMET, 2014).

Figure 1. A - Location of the Picos de Europa National Park in the area of the karst areas of the Cantabrian Mountains (the pink tone corresponds to the karst areas). B -Relief, river network and National Park climate graphs made from data from 8 weather stations of the Global Change Monitoring Network in National Parks (MAGRAMA, 2014) and 9 stations of the Spanish Meteorological Agency (AEMET, 2014).

La red fluvial presenta una baja densidad $(0,3$ $\mathrm{km} / \mathrm{km}^{2}$ ) y está formada por tres ríos principales: el Sella, el Deva y su tributario, el Cares, junto con pequeños afluentes, siendo la componente de flujo principal hacia el norte. Estos ríos se localizan a cotas de entre 100 y 1.000 m s.n.m., destacando la existencia de cañones fluviales con profundidades de hasta $2.000 \mathrm{~m}$, como el Desfiladero de los Beyos, la 
Garganta del Cares (Figura 2) o el Desfiladero de la Hermida. Los caudales estimados entre 1 y $80 \mathrm{~m}^{3} / \mathrm{s}$ proceden, en gran parte, de la descarga de los acuíferos kársticos (60-90\%) y, en menor cuantía, de la escorrentía superficial (10-40\%). Por ejemplo, en el río Cares, en época de estiaje, aproximadamente, el $60 \%$ del agua que circula procede de aguas subterráneas y el $40 \%$ restante, de la escorrentía superficial. Entre los cursos fluviales secundarios, están los ríos Covadonga, Dobra, Casaño, Duje y Urdón, con caudales inferiores a los $50 \mathrm{~m}^{3} / \mathrm{s}$. Estos ríos tienen su origen en surgencias kársticas por lo que presentan variaciones de caudal y temperatura mayores que los ríos principales (Barquín et al., 2015). El régimen de estos cursos fluviales corresponde al de ríos de montaña, que experimentan rápidas e intensas avenidas (Marquinez y Adrados, 2000), llegando a aumentar de menos de $50 \mathrm{~m}^{3} / \mathrm{s}$ a más de $500 \mathrm{~m}^{3} / \mathrm{s}$ (caudal medio horario) en menos de 7 días, debido también a la influencia nival (Deval et al., 2011).

Desde el punto de vista geológico, el Parque Nacional se incluye en dos unidades geológicas de la Zona Cantábrica: la Unidad Bodón-Ponga y la del Pisuerga-Carrión (Figura 4) (Alonso et al., 2009b). Las rocas de la Unidad Bodón-Ponga comprenden el sustrato del área central y norte del Parque Nacional (macizos Occidental, Central y Oriental de los Picos de Europa) e incluyen principalmente calizas carboní-

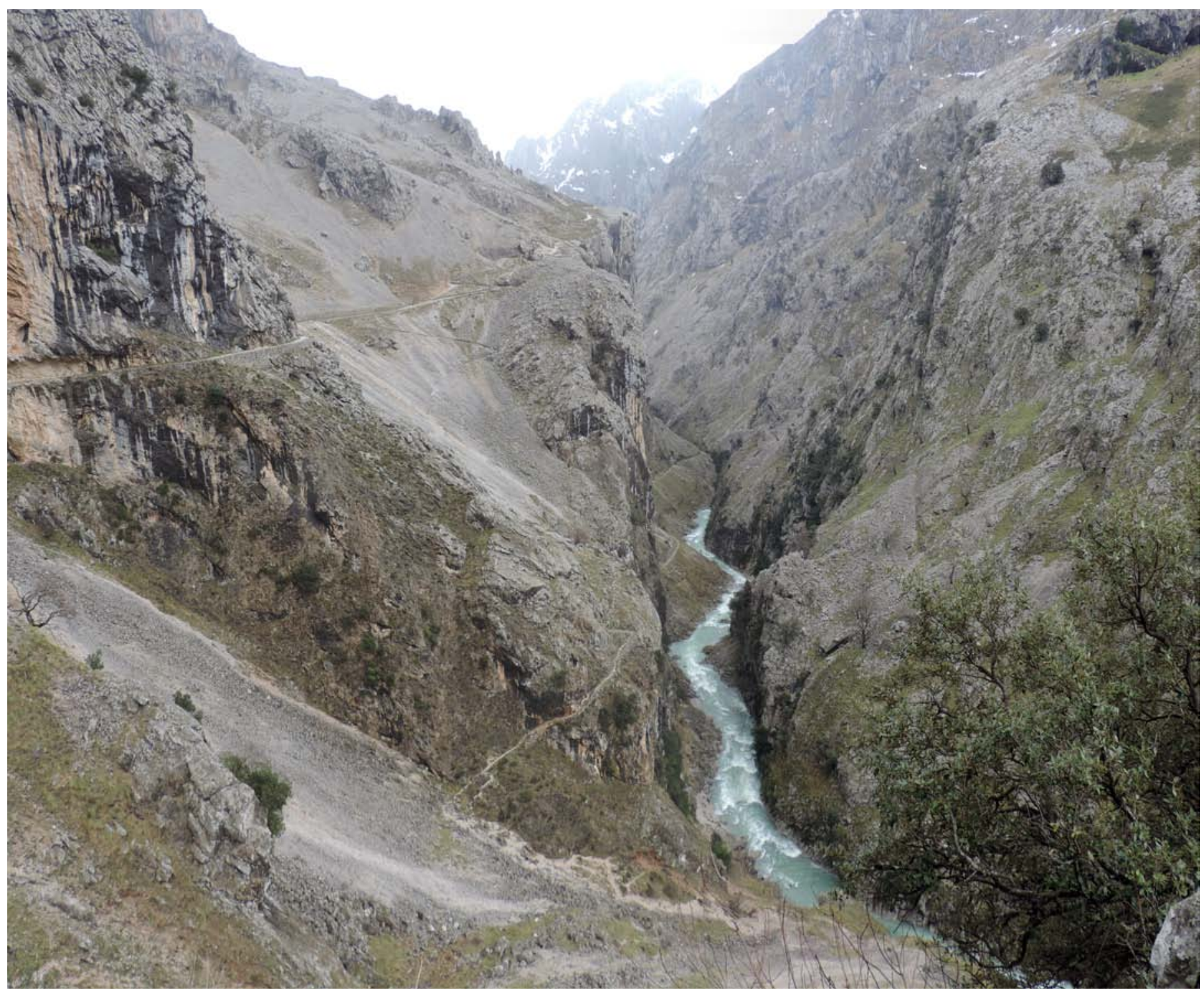

Figura 2. Vista general del desfiladero del Cares hacia el E desde la canal del Escalero; se aprecia el fuerte encajamiento del río Cares. Figure 2. General view of the Cares Gorge to the east from the Escalero Canal, showing the strong engagement of the Cares River. 
feras $y$, de forma secundaria, areniscas y lutitas del Ordovícico, Estefaniense, Pérmico y, Triásico (Bahamonde et al., 2007, López-Gómez et al., 2019). La Unidad del Pisuerga-Carrión aflora al S del Parque, en las cabeceras de los ríos Cares y Deva. La litología de esta zona es fundamentalmente siliciclástica y corresponde a areniscas, lutitas, conglomerados, y algunas calizas de edad carbonífera (Rodríguez Fernández y Heredia, 1987). Sobre estas unidades, en dos sectores (Sotres-Tresviso y Desfiladero de los Beyos) se disponen rocas pertenecientes a la Cobertera Alpina, que incluyen fundamentalmente conglomerados, areniscas y lutitas de tonos rojizos y edades del Pérmico inferior y Triásico Medio y Superior (López-Gómez et al., 2019) (Figura 4).

La estructura geológica de los Picos de Europa es compleja ya que resulta de la superposición de dos orogenias, la Varisca y la Alpina, separadas por un período extensional durante el Pérmico y Mesozoico (Merino-Tomé et al., 2009). A finales del Carbonífero se desarrolló apretado un sistema imbricado de cabalgamientos dirigidos hacia el S-SSE (Farias y Heredia, 1994), que dio lugar a un acortamiento cercano al 70\% (Merino et al., 2009). Estos cabalgamientos presentan buzamientos que van desde los $30-40^{\circ}$ en el $\mathrm{S}$ hasta verticales o incluso invertidos en el $\mathrm{N}$ con presencia de retrocabalgamientos (inclinados hacia el S y con transporte tectónico hacia el N) en el Macizo Occidental (Figura 4). Las estructuras variscas convergen en un cabalgamiento basal inclinado hacia el $\mathrm{N}$ que dispone la unidad Bodón-Ponga sobre la del Pisuerga-Carrión. Esta estructura fue modificada pos-

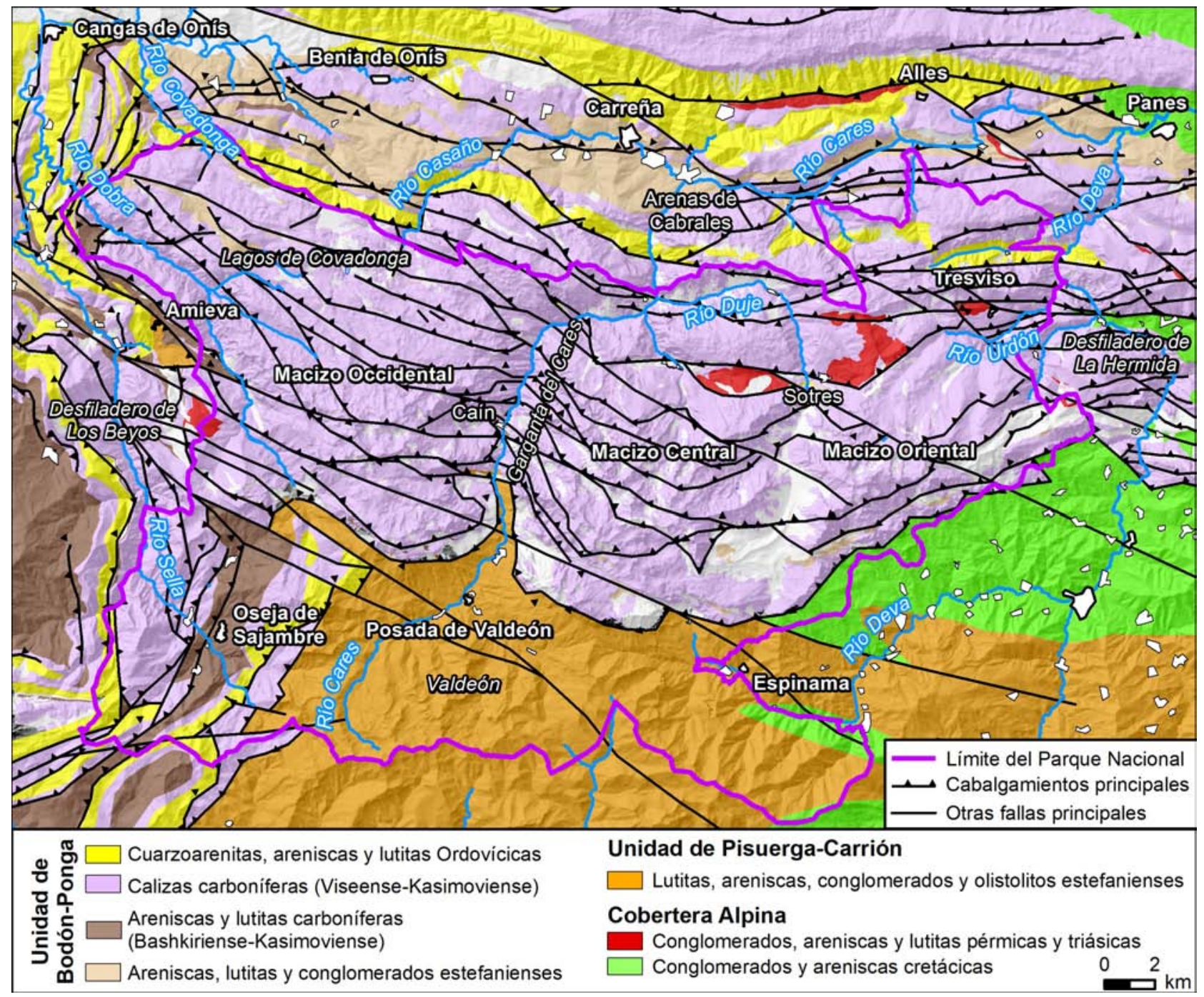

Figura 3. Mapa geológico del Parque Nacional de los Picos de Europa (modificado de Merino-Tomé et al., 2013a, 2013b).

Figure 3. Geological map of the Picos de Europa National Park (modified from Merino-Tomé et al., 2013a, 2013b). 
teriormente por el Ciclo Alpino, que comenzó por un periodo extensional permo-mesoszoico seguido por la orogenia alpina, que durante el Cenozoico (Eoceno superior-Oligoceo) desarrolló un nuevo sistema de cabalgamientos inclinado al N, cuyo nivel de despegue principal está por debajo de la estructura varisca descrita anteriormente, a la vez que se reactivaron y rotaron las fallas previas, produciéndose también la inversión de algunas de las fallas extensionales permo-mesozoicas (Alonso et al., 1996).

La presencia de los cabalgamientos variscos anteriormente citados, el gran número de estos y su superposición, ha condicionado que los Picos de Europa presenten una acumulación vertical de calizas carboníferas que llega a superar los $4 \mathrm{~km}$, lo cual, junto al fuerte encajamiento de la red fluvial y al predominio de un clima húmedo durante gran parte de su historia geológica, han propiciado un gran desarrollo del karst, y con ello, de un conjunto de acuíferos kársticos que serán mencionados más adelante. Este karst es un referente a nivel mundial ya que en él se encuentra el $14 \%$ de las simas más profundas del planeta, incluyendo también más de 3.700 cavidades conocidas con un total de $405 \mathrm{~km}$ de conductos subterráneos, en los cuales los equipos espeleológicos han identificado cientos de cursos de aguas subterráneas (Ballesteros et al., 2015a). Por encima de los 600-1.000 m s.n.m., los Picos de Europa presentan numerosas formas glaciares desarrolladas en diferentes etapas del Pleistoceno Medio y Superior, incluyendo importantes acumulaciones de till, a las que se asocian lagos de montaña (Jiménez-Sánchez et al., 2013; Serrano et al., 2013; Ruiz-Fernández et al., 2016). Así, la recarga y el desarrollo del acuífero kárstico debió estar condicionado por la dinámica glaciar, la evolución de la karstificación, la erosión de los acuitardos y los fenómenos de obstrucción de las surgencias kársticas (Ballesteros et al., 2017).

\section{Síntesis hidrogeológica del PNPE: estado del conoci- miento}

\section{Cuencas hidrográficas y masas de aguas subterráne- as}

El Parque Nacional de los Picos de Europa comprende parte de las cuencas de los ríos Sella, con 1.284 $\mathrm{km}^{2}$ de extensión y Deva, de $1.202 \mathrm{~km}^{2}$ de superficie. Ambas cuencas pertenecen a la Demarcación Hidrográfica del Cantábrico.

En lo que respecta a la delimitación de "masas de agua subterránea" (MASb), siguiendo los criterios establecidos en la Directiva Marco del Agua
(2000/60/CE), en el Parque Nacional se incluyen la MASb 012.214 Picos de Europa-Panes y la MASb 012.218 Alto Deva-Alto Cares (MIMAM, 2005; Real Decreto 399/2013, BOE $n^{\circ} 137$, de 8 de junio). La primera masa comprende $883 \mathrm{~km}^{2}$ de la superficie situada al $\mathrm{N}$ y centro de los Picos de Europa, mientras que la segunda abarca $296 \mathrm{~km}^{2}$, localizados en la vertiente sur del Parque. En el primer caso, predominan los acuíferos kársticos de naturaleza calcáreo-dolomítica, desarrollados en las calizas carboníferas (IGME, 1984; López Geta et al., 1997), mientras que en el segundo, las litologías presentes comprenden materiales paleozoicos que incluyen lutitas, areniscas, conglomerados, cuarzoarenitas y pizarras (Plan Hidrológico de la Demarcación Hidrográfica del Cantábrico Occidental 2009-2013, aprobado según Real Decreto 399/2013).

\section{Clasificación hidrogeológica de las rocas y sedimen- tos del PNPE}

A escala regional, considerando las características de permeabilidad de los materiales, en el Parque Nacional de los Picos de Europa, las formaciones presentes se pueden clasificar como acuíferos o acuitardos. El acuífero principal es de naturaleza kárstica, constituido por materiales carbonatados carboníferos, mientras que el resto de los materiales intercalados o situados a muro o techo del acuífero son de naturaleza detrítica y funcionan como acuitardos. Desde el punto de vista estratigráfico, de muro a techo, se pueden diferenciar, utilizando los criterios de caracterización hidrogeológica y edad de cada unidad, los siguientes acuíferos y acuitardos:

Acuitardo del Paleozoico Inferior. Está formado por areniscas, en gran parte, cuarzoarenitas y lutitas del Ordovícico y Devónico, correspondientes a las formaciones Oville, Barrios, Ermita y Vegamián/Baleas, fundamentalmente, que presenta un espesor máximo de unos $1.000 \mathrm{~m}$. Estos materiales afloran de forma mayoritaria en el $\mathrm{N}$ de los Picos de Europa y funcionan, en conjunto, como acuitardo, ya que, aunque han desarrollado cierta permeabilidad por fisuración, este parámetro toma, en términos generales, valores bajos, del orden de $10^{-2}$ a $10^{-4} \mathrm{~m} / \mathrm{d}$ (Herguedas et al., 2009). Cabe mencionar, que, en otras áreas de la Cordillera Cantábrica, estas cuarzoarenitas aparecen alteradas o muy fracturadas, constituyendo acuíferos porosos secundarios llegando a alcanzar valores de conductividad hidráulica entre $10^{2}$ y $10^{3} \mathrm{~m} /$ día (Alonso et al., 2009a, Herguedas et al., 2009, Garrido Ruiz et al., 2009; Valenzuela et al., 2015), por lo que no se descarta que pudiera suceder lo mismo en algún sector de los Picos de Europa. A muro de la formación 
Oville, se encuentra la formación calcárea Láncara, en la que se ha desarrollado una permeabilidad secundaria por karstificación más elevada que la de las formaciones siliciclásticas, si bien, debido a su reducido espesor, se ha agrupado con las formaciones Oville y Barrios (Figura 4).

Acuífero kárstico carbonífero. Está constituido por una serie de más de $1.500 \mathrm{~m}$ de calizas carboníferas correspondientes a las formaciones Alba, Barcaliente, Valdeteja y Picos de Europa (Viseense-Moscoviense) que afloran en la mayor parte de los Picos de Europa y representan el acuífero principal. Estas formaciones presentan un elevado desarrollo kárstico, evidenciado por la casi ausencia de cursos de aguas superficiales, la existencia de cuencas endorreicas y por la gran circulación del agua subterránea a través de conductos kársticos (Pulido, 2014); además, se pone de manifiesto en presencia de más de $400 \mathrm{~km}$ de conductos documentados (Ruiz-Fernández y Poblete Piedrabuena, 2012; Ballesteros et al., 2015a). A techo, se encuentran las formaciones Las Llacerias y Demués, predominantemente de composición carbonatada, que por su reducido espesor y comportamiento hidrogeológico análogo se consideran también parte de esta unidad. El sistema se recarga a través de las precipitaciones que, tanto en forma de agua como de nieve, alcanzan los materiales permeables; la descarga tiene lugar a través de numerosas surgencias, ubicadas tanto en el Parque como en zonas aledañas (Figura 4).

Acuitardo de la secuencia del Carbonífero Superior. Está formado por una sucesión de calizas con chert y lutitas correspondientes al dominio estratigráfico de base de talud y cuenca de las formaciones Valdeteja y Picos de Europa (Bahamonde et al., 2007). Estos materiales presentan importantes variaciones laterales de espesor que oscilan desde unos $120 \mathrm{~m}$ en la zona S (Canal de Pedavejo, Bahamonde et al., 2007) hasta llegar a desaparecer en determinadas ubicaciones, principalmente en el sector norte del Parque. Debido a la complejidad estructural, afloran intercalados dentro del acuífero kárstico principal. Desde el punto de vista hidrogeológico, su carácter de acuitardo puede llegar a condicionar la ubicación de desarrollos kársticos (Torca del Cueto los Senderos, Smart, 1985) (Figura 4).

Acuitardo carbonífero sinorogénico. Con un espesor de unos $500 \mathrm{~m}$, está formado por areniscas, lutitas, conglomerados y calizas estefanienses de la unidad de la Bodón-Ponga (formaciones Gamonedo, Lebeña, Áliva, Cavandi y Puentellés). Los valores de permeabilidad, atendiendo a sus características litológicas y estructurales se estiman de bajos a muy bajos, si bien los niveles calcáreos, cuando se encuentran karstificados pueden constituir niveles acuíferos de escasa entidad. En conjunto, estos materiales aunque se disponen discordantemente sobre la serie carbonatada carbonífera, pueden aparecer cobijados por las láminas cabalgantes que contienen la serie carbonatada carbonífera, constituyendo barreras de permeabilidad dentro del acuífero kárstico principal (Figura 4).

Acuitardo carbonífero detrítico. Constituido por lutitas, areniscas conglomerados brechas calcáreas y calizas de la Unidad Pisuerga-Carrión (grupos Potes, Pando, Maraña-Brañas, Pontón, Valdeón y Remoña). Este conjunto, que sólo aflora al $\mathrm{S}$ de Parque, puede alcanzar un espesor máximo de $3.500 \mathrm{~m}$ (Adrados et al., 2010). Estos materiales constituyen el límite inferior del acuífero kárstico, como consecuencia de la superposición que se produjo mediante el ya mencionado cabalgamiento basal. En conjunto, la permeabilidad desarrollada por fracturación y fisuración, puede estimarse como baja a muy baja, si bien los materiales calcáreos (calizas y brechas calcáreas) pueden presentar valores más elevados debido a procesos de karstificación (Figura 6).

Acuitardo cuaternario. Está formado por sedimentos superficiales, en su mayor parte, de grano fino, que, en ocasiones, pueden incluir cantos y bloques. Predominan los depósitos de origen glaciar, y en menor medida, también se encuentran de gravedad y kársticos. Se localizan tanto al $\mathrm{S}$ como al norte de los Picos de Europa, entre las cotas 600-1.300 m s.n.m, preferentemente. Son considerados materiales de baja permeabilidad debido a su granulometría, la abundancia de zonas palustres desarrolladas sobre ellos y el hecho de que en lugares de contacto con el acuífero kárstico principal surjan manantiales kársticos, sobre todo, en el NO del Macizo Occidental (Figuras 4 y 5 ).

Acuífero cuaternario. Lo constituyen los depósitos superficiales cuyo origen está en procesos de laderas, muy activos en toda la región, o en procesos fluviales, restringidos a los cauces de los ríos. Los materiales predominantes son cantos y bloques, con escasa o nula matriz. Su elevada macroporosidad permite clasificarlos como acuíferos, en cuyo límite inferior afloran, en múltiples casos, pequeños manantiales estacionales (Figuras 4 y 5 ).

\section{Geometría del acuífero kárstico}

El acuífero kárstico presenta una geometría compleja (Figuras 6 y 7), condicionada por la presencia de acuitardos que actúan como barreras de permeabilidad y compartimentan hidráulicamente el acuífero, 


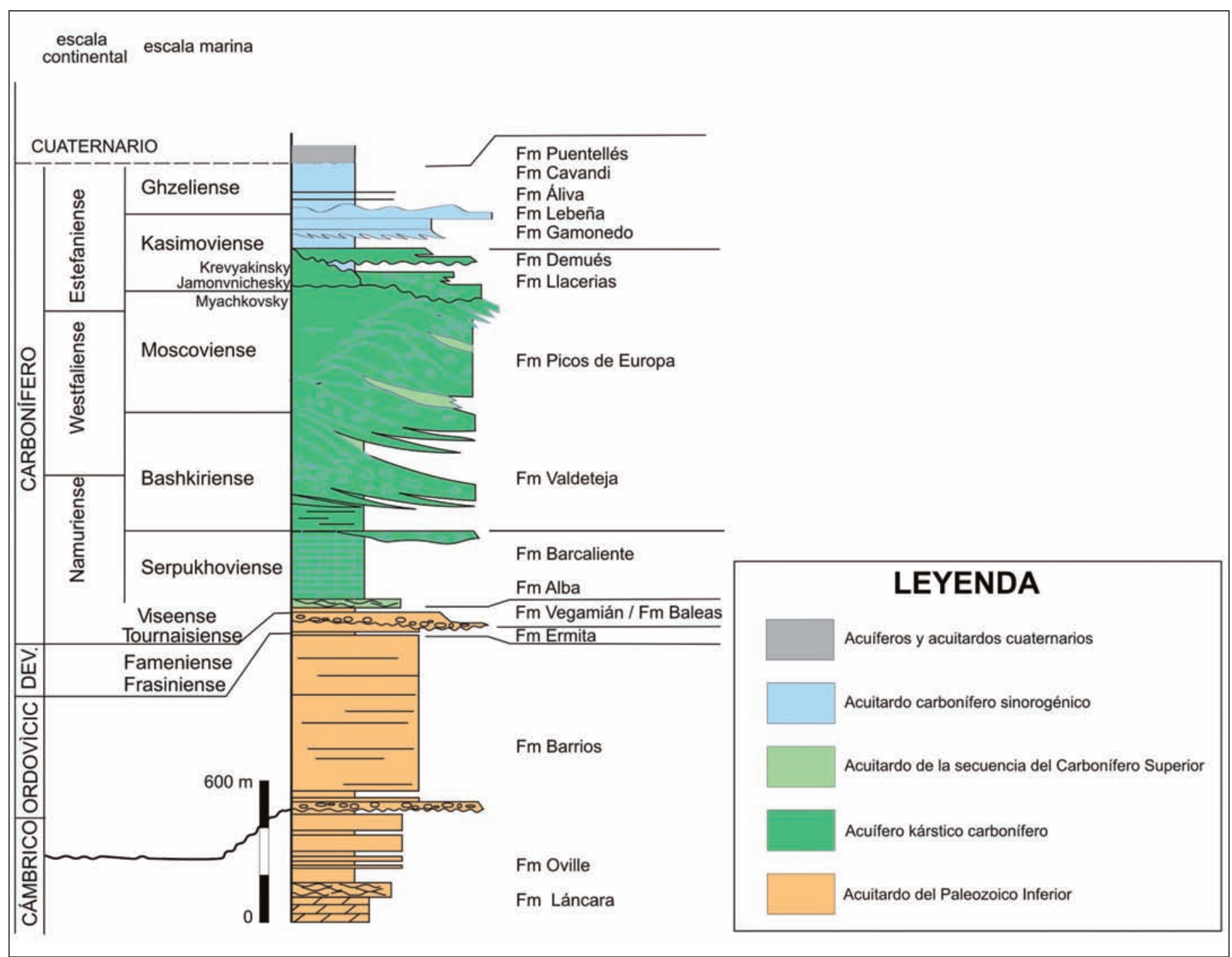

Figura 4. Columna hidrogeológica sintética de la Unidad Bodón-Ponga en los Picos de Europa (Modificada de Bahamonde et al. 2007). Figure 4. Synthetic hydrogeological column of the Bodon-Ponga Unit in the Picos de Europa (Modified from Bahamonde et al., 2007).

individualizándolo en unidades menores con superficies piezométricas situadas a diferentes cotas que, en ocasiones, quedan desconectadas de los ríos que marcan el nivel basal (Arquer Prendes-Pando, 2001). Su orientación general es O-E y está dividido en compartimentos o sectores independizados total o parcialmente denominados "cuerpos de agua subterránea menores" (en adelante SGWB, acrónimo de la terminología inglesa, "smaller groundwater bodies") de tamaños comprendidos entre 2 y $108 \mathrm{~km}^{3}$ de volumen (Ballesteros et al., 2013, 2015c). En ellos, el espesor de la zona saturada es, en general, menor que el de la zona no saturada, que puede alcanzar los 2.300 m (Ballesteros et al., 2015; Ballesteros, 2016). La compartimentación del acuífero kárstico se debe a la pre- sencia de formaciones de baja o muy baja permeabilidad (acuitardos) que actúan como barreras de permeabilidad, condicionando la existencia de sectores parcialmente independizados cuyos volúmenes son variables (se han descrito valores para la zona saturada entre 0,01 y $60 \mathrm{~km}^{3}$, Ballesteros et al., 2013, 2015c). El borde impermeable basal lo constituyen los materiales de la Unidad de Pisuerga-Carrión, que se disponen, por contacto mecánico, bajo el acuífero kárstico, coincidiendo el contacto entre ambos con el nivel de despegue de los cabalgamientos variscos. En el $\mathrm{S}$, dicho límite se encuentra inclinado entre 30 y $60^{\circ} \mathrm{N}$, mientras que, en el $\mathrm{N}$, su inclinación varía entre $60^{\circ} \mathrm{N}$, verticalizándose en profundidad. (Figuras 6 y 7$)$. 


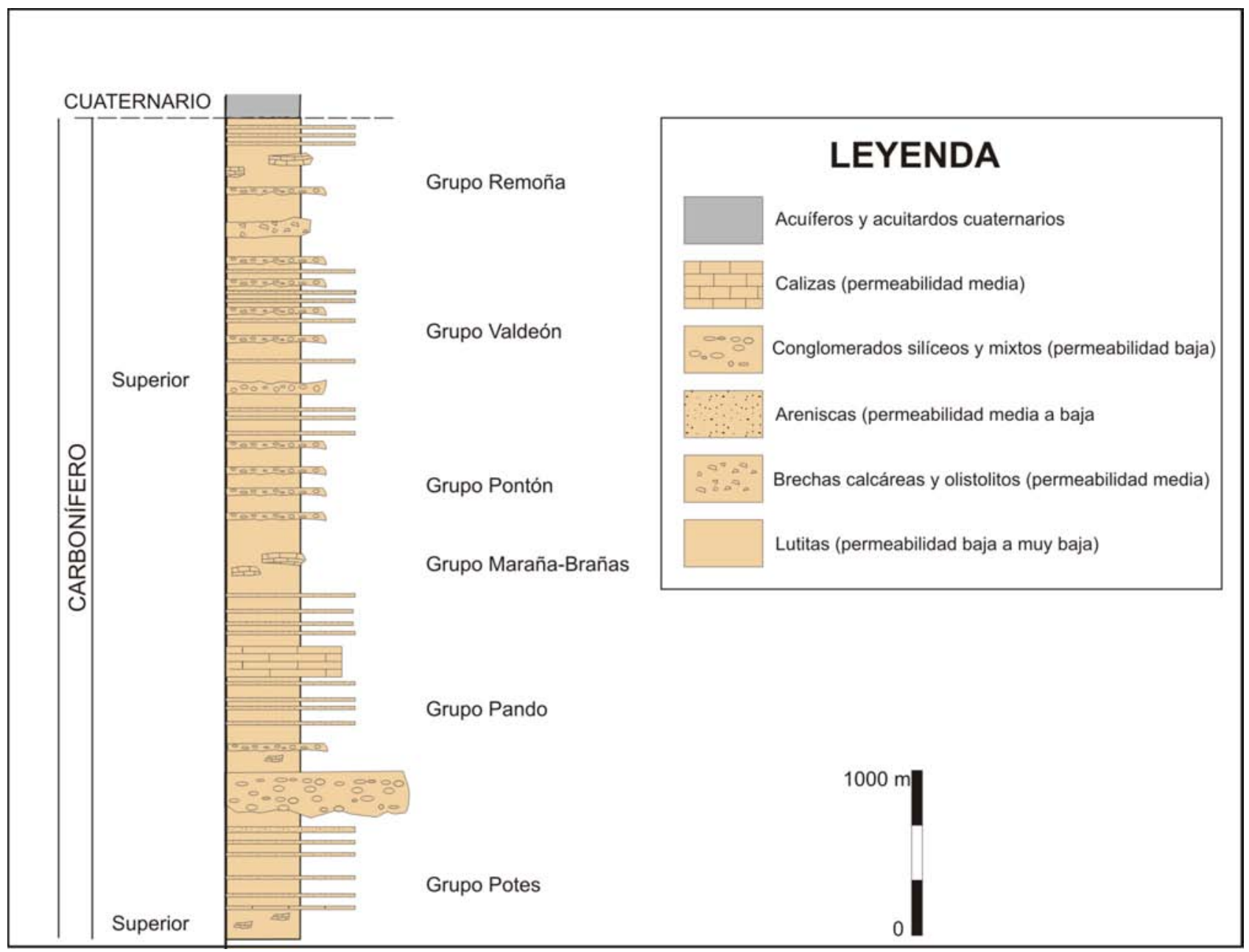

Figura 5. Columna hidrogeológica sintética del acuitardo carbonífero detrítico (Unidad Pisuerga-Carrión) (Modificada de Adrados et al, 2010).

Figure 5. Synthetic hydrogeological column of the detritic carboniferous aquitard (Pisuerga-Carrión Unit) (Modified by Adrados et al., 2010).

\section{Hidrodinámica}

El acuífero kárstico se comporta en su mayor parte como acuífero libre, si bien, en determinadas situaciones, como consecuencia de inversiones tectónicas de la serie, se dispone por debajo de los niveles de acuitardos mencionados anteriormente, y podría funcionar como acuífero semiconfinado (Figuras 6 y 7 ).

La recarga del acuífero se realiza, preferentemente, por infiltración directa de la lluvia o la fusión del manto nival (Miotke 1968; Firman 1979) a través del exokarst. Se ha estimado un coeficiente de infiltración que puede oscilar entre el 60 y el $100 \%$ de la lluvia útil para el conjunto de los Picos de Europa, en función del grado de recubrimiento superficial (IGME, 1982). Y por tanto, considerando valores de precipitación entre 1.200 y 1.700 mm (AEMET, periodo 2013-
2016), el volumen estimado para la infiltración directa oscila entre 530 y $750 \mathrm{hm}^{3} /$ año. Frecuentemente, los eventos de precipitación son intensos y la fusión del manto nival es muy rápida debido a subidas bruscas de la temperatura, muchas veces causadas por el efecto Föehn (Fernández García y Rasilla, 1992). Estos fenómenos provocan variaciones muy rápidas en la recarga del acuífero, con desfases muy reducidos entre la entrada de precipitación y la respuesta en el aumento de caudal en manantiales. Puntualmente, también puede existir cierta entrada de agua en el acuífero kárstico a través de sumideros kársticos y de tramos de ríos influentes. $Y$ además, en algunos casos, podría haber conexión hídrica entre los SGWBs situados a diferentes cotas, de tal forma que los de mayor altitud recargarían a los de menor cota topográfica. 


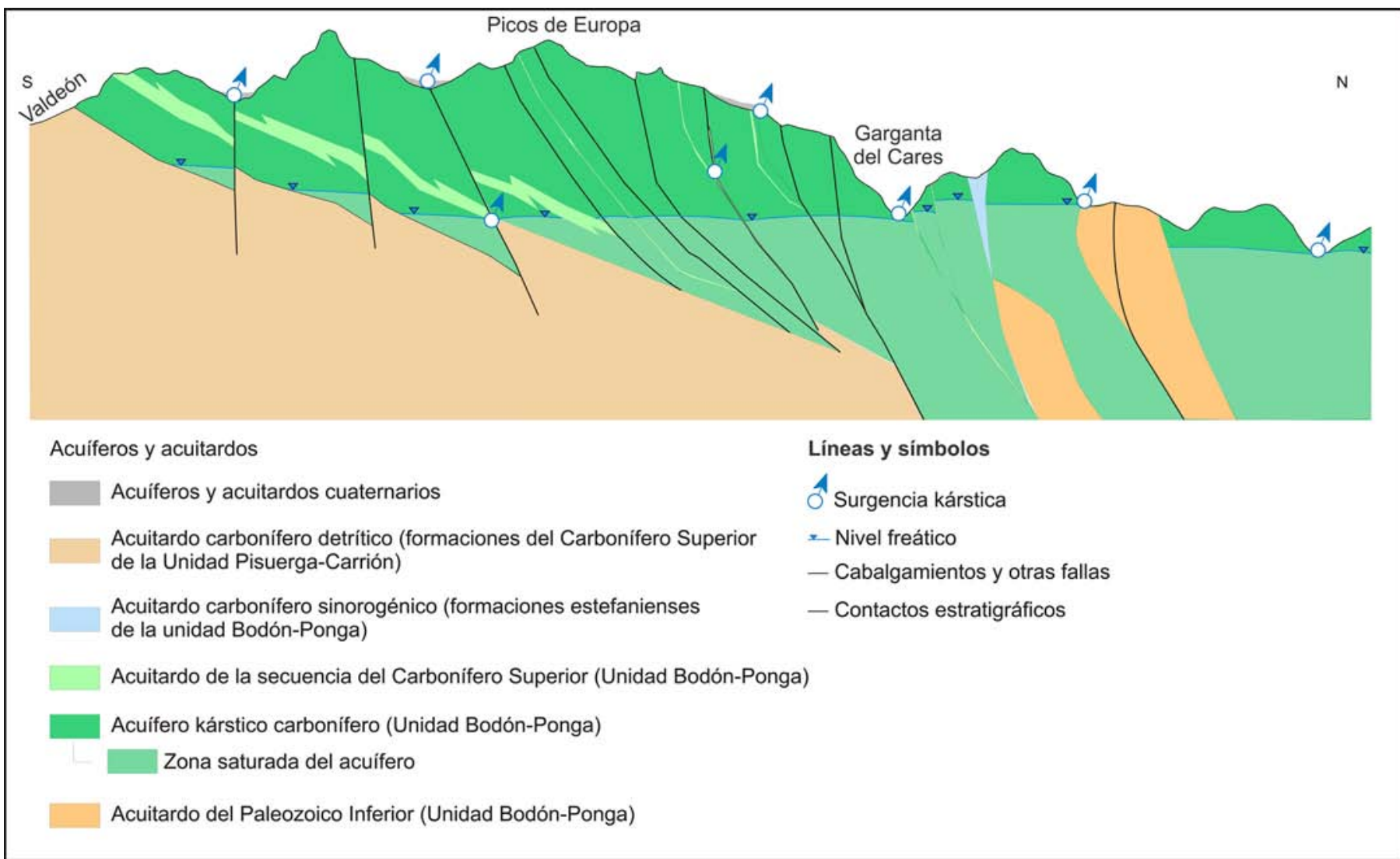

Figura 6. Corte hidrogeológico esquemático de dirección S-N en el que se muestra la disposición de los materiales, clasificados según la leyenda hidrogeológica establecida por IGS, IAHS, IAH y UNESCO 1970 (Struckmeier y Margat, 1995) y los niveles piezométricos del acuífero kárstico. (Modificado de Ballesteros et al., 2013, 2015b).

Figure 6. Schematic hydrogeological section of S-N direction showing the disposition of the materials, classified according to the hydrogeological legend established by IGS, IAHS, IAH and UNESCO 1970 (Struckmeier and Margat, 1995), and the piezometric levels of the karst aquifer (after Ballesteros et al., 2013, 2015b).

Los sumideros constituyen drenajes que concentran el agua de arroyos, pequeños manantiales o de flujos asociados a formaciones superficiales, con caudales generalmente menores de $4 \mathrm{~L} / \mathrm{s}$. Como ejemplos están los arroyos situados al $\mathrm{N}$ de Peña Hascal y el de la Vega de Comeya, que en ambos casos, se sumen en el karst después de unos $1.500 \mathrm{~m}$ de recorrido en superficie.

Los cursos fluviales más significativos son los ríos Güeña, Dobra, Casaño, Cares, Duje, Pomperi y La Beyera (Figura 7). Se han reconocido tramos influentes en los ríos Cares, Duje y Pomperi y tramos efluentes en los ríos Güeña, Deva, Urdón y Cares (Figura 7). En el caso de los tramos influentes, la diferencia estimada entre la cota a la que discurre el río y la del nivel piezométrico es del orden de $20 \mathrm{~m}$ en los ríos Cares y Duje, pudiendo alcanzar los $80 \mathrm{~m}$ en el tramo superior del río Pomperi. Cabe destacar, en este último caso, que el agua, después de infiltrarse en el acuífero, aflora de nuevo en la surgencia de El Burdiu, situada aguas abajo en el mismo valle del río Pomperi, lo que se ha visto corroborado mediante un ensayo de trazadores realizado por grupos espeleológicos (G.E. Diaño Burlón y A.D. Cuasacas, 2011).

El flujo de agua subterránea tiene lugar, en su mayor parte, a través de conductos kársticos bien desarrollados. La presencia de los acuitardos anteriormente mencionados funcionan como barreras subterráneas (Arquer Prendes-Pando 2001), condicionando en parte la circulación del agua subterránea y, por tanto, la evolución de la karstificación y la dirección general del flujo. La inclinación general de las estructuras hacia el $\mathrm{N}$ propicia que el sentido del flujo subterráneo regional se oriente hacia el $\mathrm{N}$, aspecto que se pone de manifiesto en la localización de las principales surgencias kársticas en la parte septentrional de los Picos de Europa. No obstante, a escala local, el flujo subterráneo puede circular en direccio- 


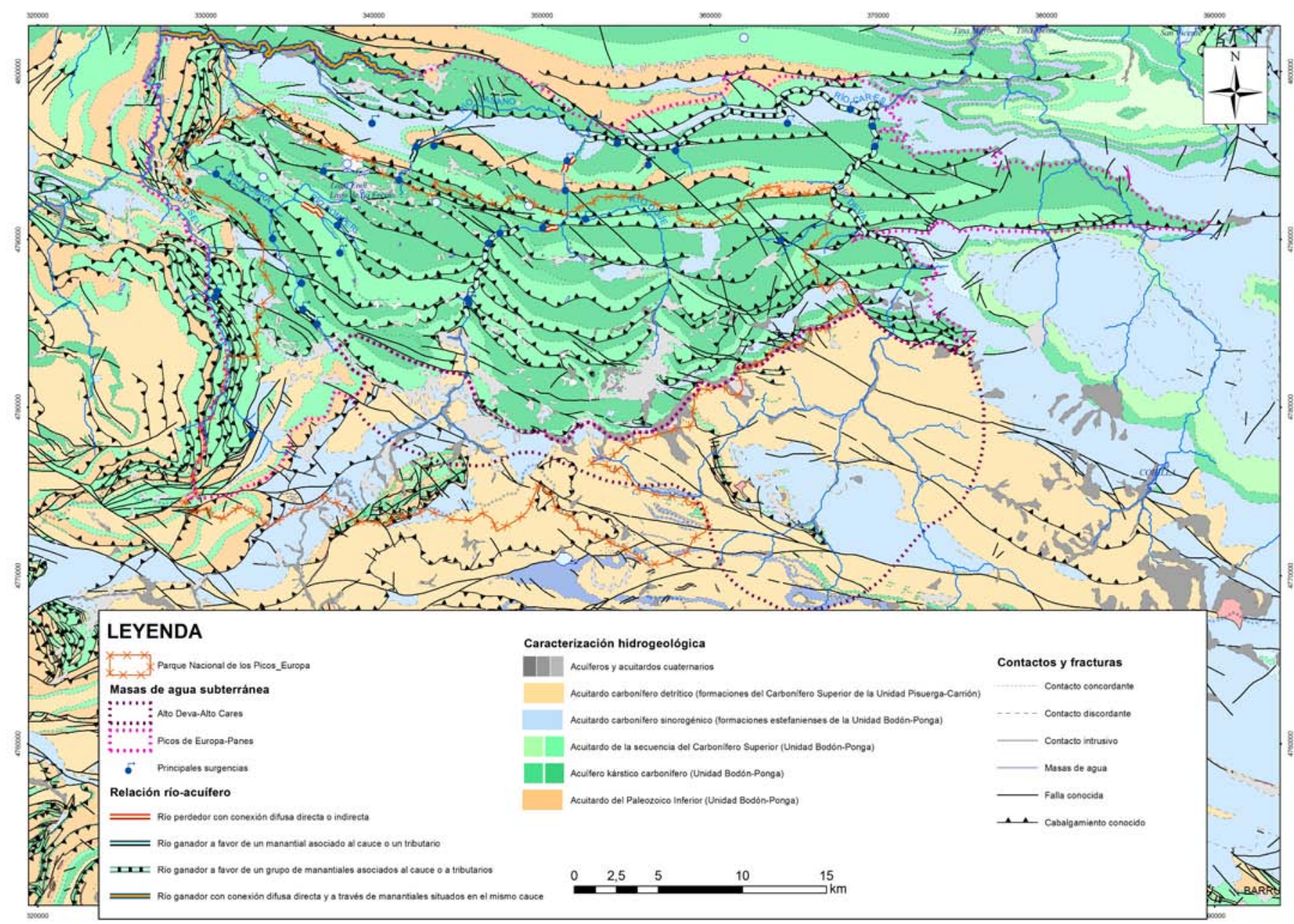

Figura 7. Mapa hidrogeológico del Parque Nacional de los Picos de Europa (modificado de IGME, 2007 e IGME-DGA, 2009), con situación de tramos efluentes e influentes clasificados según la leyenda hidrogeológica establecida por IGS, IAHS, IAH y UNESCO 1970 (Struckmeier and Margat, 1995).

Figure 7. Hydrogeological map of the Picos de Europa National Park (modified from IGME, 2007 and IGME-DGA, 2009), with situation of effluent and influent sections classified according to the hydrogeological legend established by IGS, IAHS, IAH and UNESCO 1970 (Struckmeier and Margat, 1995).

nes diversas, como lo evidencian los ensayos de trazadores realizados por distintos equipos espeleológicos (Firman, 1979; Fabre y Fabriol, 1984; Collignon, 1986; Lloyd, 1990; Herrero, 1992), que establecieron trayectorias de flujo hacia el N, NO, NE y E (Tabla 1). Estas trayectorias son, generalmente, paralelas a las direcciones de estratificación y cabalgamientos si bien, en ocasiones están controladas por fallas de dirección N-S; un ejemplo significativo es la surgencia de Juansabeli.

Los ensayos de trazadores (Tabla 1) están descritos en la documentación espeleológica generada entre los años 1967 y 2015. Estos ensayos consistieron en inyecciones de entre 0,1 y $10 \mathrm{~kg}$ de fluoresceína sódica o rodamina $B$ en cursos de aguas subterrá- neas, generalmente en etapas de aguas bajas. En el $90 \%$ de los casos se detalla el tiempo transcurrido entre el vertido del trazador y el momento en que se detecta su presencia. En un $43 \%$ de estos ensayos, los tiempos, se cifran entre 3 y 30 horas para el recorrido de distancias de $300 \mathrm{~m}$ a $2 \mathrm{~km}$ entre el lugar de inyección del trazador y la surgencia kárstica, infiriéndose velocidades de entre 70 y $380 \mathrm{~m} / \mathrm{h}$ para las aguas subterráneas, algo inferiores a los valores $(300-500 \mathrm{~m} / \mathrm{h})$ calculados para zonas de karst alpinos (Ford, 1993). En el $57 \%$ restante de los casos, se registraron tiempos de entre 2 y 20 días para distancias de entre 300 $\mathrm{m}$ y $8 \mathrm{~km}$, estimándose velocidades de flujo subterráneo entre 6 y $17 \mathrm{~m} / \mathrm{h}$. Estos valores son muy bajos en comparación con los datos obtenidos en otras áreas 
Mónica Meléndez, et al., 2019. Hidrogeología del Parque Nacional de los Picos de Europa... Boletín Geológico y Minero, 130 (4): $593-614$

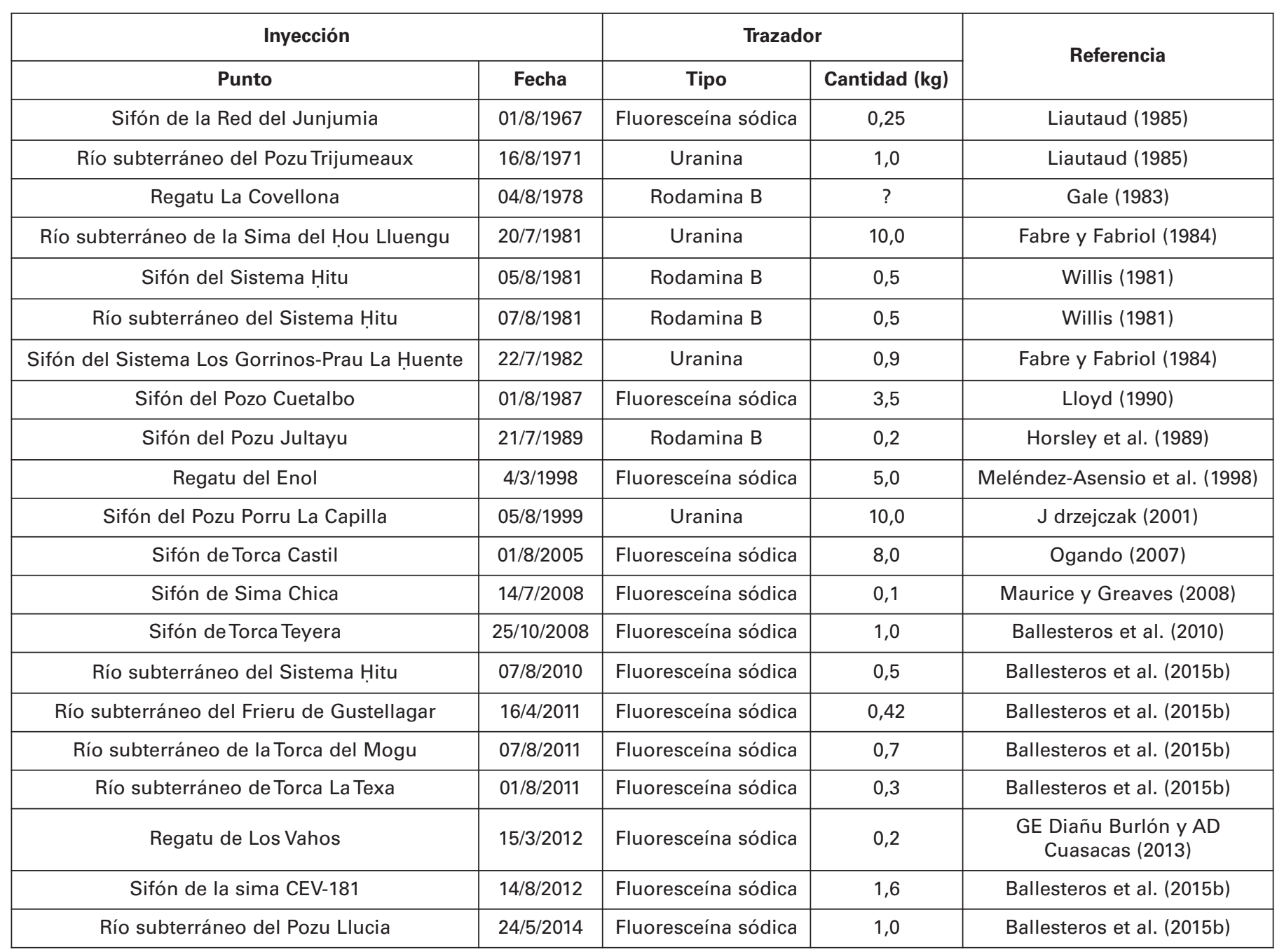

Tabla 1. Ensayos de trazadores realizados por los equipos espeleológicos, organizados cronológicamente según la fecha de inyección. Table 1. Tracer tests performed by the speleological teams, organized chronologically according to the injection date.

kársticas kárstica (p.e. Goldscheider et al. 2008; Karami y Ashjari 2010), por lo que serán objeto de investigaciones posteriores.

La descarga del acuífero se realiza prioritariamente a través de las más de 20 surgencias situadas, en su mayor parte, en valles fluviales en las proximidades de los cauces (Firman 1979; Virgós Soriano et al. 1980; Fabre y Fabriol 1984; Collignon 1986; Lloyd 1990; López Geta et al. 1997). La Tabla 2 muestra las surgencias principales, si bien, se debe tener en consideración que a través de una misma surgencia (o varias surgencias asociadas) se drenan conjuntamente las aguas superficiales y subterráneas correspondientes a una superficie del terreno que puede comprender uno o varios compartimentos del acuífero kárstico. Por lo general, se ha podido observar que estas áreas drenadas presentan una disposición alar- gada siguiendo la dirección O-E y su superficie oscila entre 1 y $100 \mathrm{~km}^{2}$. Las surgencias constituyen los principales aportes a los ríos Casaño, La Beyera, Pomperi y Hunhumia, así como a otros de naturaleza similar.

Los caudales drenados a través de las surgencias están estrechamente relacionado con la extensión del área de recarga de cada una de ellas, estimándose una descarga anual de $3 \pm 1 \mathrm{hm}^{3}$ por cada $\mathrm{km}^{2}$ de superficie drenada (Figura 9). Los caudales mínimos medidos llegan a superar los $500 \mathrm{l} / \mathrm{s}$ en las principales surgencias (Los Molinos, La Fuente del Nacimiento y el Farfao de la Viña, Tabla 2). Cabe destacar que durante las etapas de crecida los caudales pueden aumentar en uno o dos órdenes de magnitud (Ballesteros et al., 2013, 2015c), lo que está relacionado con las variaciones significativas de la recarga y con la elevada transmisividad del acuífero (Virgós Soriano et al. 
Mónica Meléndez, et al., 2019. Hidrogeología del Parque Nacional de los Picos de Europa... Boletín Geológico y Minero, 130 (4): $593-614$

\begin{tabular}{|c|c|c|c|c|c|}
\hline Nombre & $\begin{array}{c}\text { Cuenca } \\
\text { hidrográfica }\end{array}$ & Situación & $\begin{array}{c}\text { Cota } \\
\text { (m s.n.m) }\end{array}$ & $\underset{(1 / s)}{O \min }$ & $\underset{(I / s)}{Q \max }$ \\
\hline Fuente del Nacimiento & Urdón/Deva & Macizo Oriental & 494 & 600 & 2100 \\
\hline Los Molinos & Cares & Macizo Central & 440 & 600 & \\
\hline Fuente Obar & Cares & Macizo Occidental & 178 & 120 & 340 \\
\hline Güeyo Reinazo & Covadonga/Sella & Macizo Occidental & 271 & 167 & - \\
\hline Las Hollas de Doñea & Cares & Macizo Occidental & 325 & 90 & 500 \\
\hline Los Brazos & Casaño/Cares & Macizo Occidental & 420 & 12 & 250 \\
\hline Las Pálvoras & Casaño/Cares & Macizo Central & 490 & 150 & 900 \\
\hline Frieru Los Vahos & Pomperi/Dobra/Sella & Macizo Occidental & 1305 & 10 & 123 \\
\hline Fuente Molín de Mildón & Cares & Peñas Cabriegas & 125 & 30 & 500 \\
\hline Fuente Juansabeli & Cares & Área de Portudera & 145 & 3 & 55 \\
\hline
\end{tabular}

Tabla 2. Manantiales principales en el Parque Nacional de Picos de Europa (Q min y Q max significan, respectivamente, "caudal mínimo" y "caudal máximo").

Table 2. Main springs in the Picos de Europa National Park ( $Q$ min and Q max mean, respectively, "minimum flow" and "maximum flow").

1980). La ubicación de los manantiales principales está condicionada por la presencia de materiales poco permeables correspondientes a los acuitardos anteriormente citados (acuitardo carbonífero detrítico en la Unidad Pisuerga-Carrión, acuitardo carbonífero sinorogénico, acuitardo de la secuencia del Carbonífero superior y acuitardo del Paleozoico Inferior en la Unidad Bodón-Ponga), así como por la situación de fallas de escala regional, probablemente alpinas, que cortan algunas estructuras previas.

La cota topográfica de descarga, atendiendo a los datos disponibles de las principales surgencias, es algo dispar en los tres macizos de los Picos de Europa. Así, en el Macizo Oriental, la Fuente del Nacimiento se sitúa a una altitud de 500 m s.n.m., en el Macizo Central, las surgencias están ubicadas entre los 315 y los 490 m s.n.m., mientras que en el Macizo Occidental la diferencia de cota entre las surgencias es elevada, variando desde los $180-260$ m s.n.m de los manantiales que descargan al río Cares, hasta los más de $1.200 \mathrm{~m}$ de los que vierten al río Pomperi. Al $\mathrm{N}$ del Macizo Central, ya fuera de los límites del Parque, en la cuenca del río Cares, los manantiales Molín de Mildón y Juansabeli, drenan el acuífero carbonatado a cotas entre 120 y 150 m s.n.m. (Tabla 2).

Las cotas de surgencia de los manantiales, así como las cotas de descarga a los cauces, ha permiti- do inferir que la superficie piezométrica se localiza a cotas entre 145 y 1.425 m s.n.m. descendiendo, en general, de forma escalonada hacia el norte, con diferencias entre niveles piezométricos del orden de 100 a $300 \mathrm{~m}$ y un valor máximo de $600 \mathrm{~m}$. Todo ello es compatible con la compartimentación del acuífero debida a la influencia de la tectónica, anteriormente mencionada.

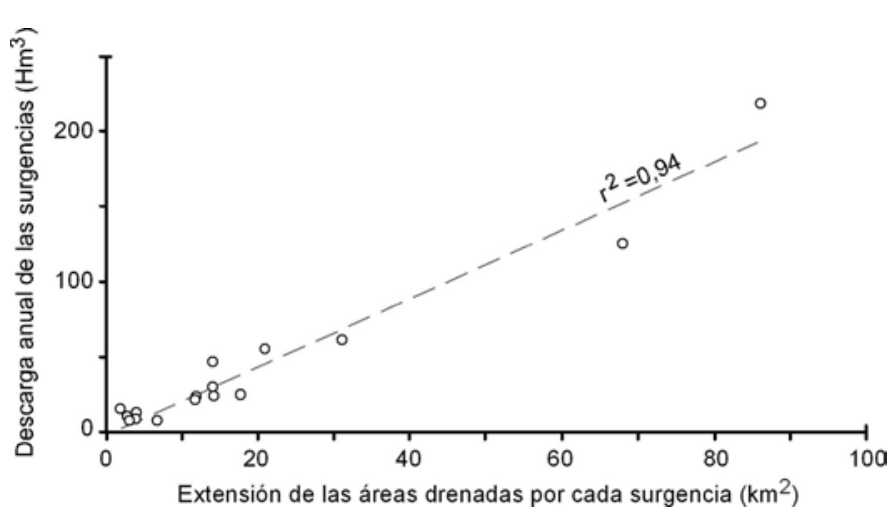

Figura 8. Relación entre la descarga anual estimada para cada surgencia y el área de recarga de cada una de ellas (datos procedentes de Ballesteros, 2016).

Figure 8. Relationship between the estimated annual discharge for each upwelling and the recharge area for each of them (data from Ballesteros, 2016). 


\section{Hidroquímica}

Las aguas kársticas de los Picos de Europa son bicarbonatadas cálcicas (Figura 9), presentan temperaturas de entre 2,5 y $11^{\circ} \mathrm{C}$, valores de $\mathrm{pH}$ comprendidos entre 7,9 y 8,9 y valores de conductividad eléctrica entre 120 y $250 \mathrm{~S} / \mathrm{cm}$, disminuyendo con el incremento de altitud. La baja concentración en aniones $(160 \pm 30 \mathrm{mg} / \mathrm{l})$ y cationes $(50 \pm 10 \mathrm{mg} / \mathrm{l})$ está condicionada por la naturaleza de la recarga y por el escaso tiempo de residencia en el karst (Miotke 1968; Virgós Soriano et al. 1980; López Geta et al. 1997; Arquer Prendes-Pando 2001). Como puede apreciarse en la Figura 9 (Ballesteros, 2006, base de datos Aguas del IGME), en el contenido aniónico predomina el bicarbonato $(150 \pm 30 \mathrm{mg} / \mathrm{l})$, seguido del cloruro $(5 \pm 4 \mathrm{mg} / \mathrm{l})$ y del sulfato $(3 \pm 1 \mathrm{mg} / \mathrm{l})$. El catión mayoritario es el calcio $(40 \pm 10 \mathrm{mg} / \mathrm{l})$, mientras que el sodio, el magnesio y el potasio aparecen en bajas concentraciones $(3 \pm 3$ $\mathrm{mg} / \mathrm{l}, 3 \pm 1 \mathrm{mg} / \mathrm{l}, \mathrm{y}>1 \mathrm{mg} / \mathrm{l}$, respectivamente). En cuanto a su distribución, el contenido en iones se reduce notablemente a medida que aumenta la altitud $y$, además, está influenciado por la presencia de las nieblas salinas procedentes del Mar Cantábrico, que aportan cloro y sodio.

Cabe destacar la presencia de aguas termales en el Parque; así, en el límite oriental, en el cauce del río Deva, se localizan varias surgencias termales, una de las cuales, con una temperatura que puede alcanzar los $60^{\circ} \mathrm{C}$, da servicio al Balneario de La Hermida. La ubicación de esta surgencia de aguas termales podría estar relacionada con estructuras tectónicas alpinas (Marquínez, 1978; García Martín, 2018). Otra surgencia con temperatura anómala, aunque no tan elevada, es Fuente Obar $\left(23^{\circ} \mathrm{C}\right)$, situada en el cauce del río Cares, si bien se considera que ambas se ajustan a un modelo de funcionamiento similar. Las aguas de origen meteórico infiltradas incrementan su temperatura en función de un gradiente geotérmico, hasta llegar a una profundidad máxima donde se produce el último equilibrio termodinámico. La fracturación generada por la Orogenia Alpina, propicia un ascenso rápido, lo que evita la perdida de calor. Desde el punto de vista hidroquímico, estas aguas son predominantemente sulfatadas-cloruradas sódicas, difiriendo notablemente de las aguas del acuífero kárstico.

\section{Utilización de las aguas subterráneas}

Las aguas subterráneas del Parque, además de constituir un soporte imprescindible para su valor ambiental y ecológico, han sido objeto de explotación desde

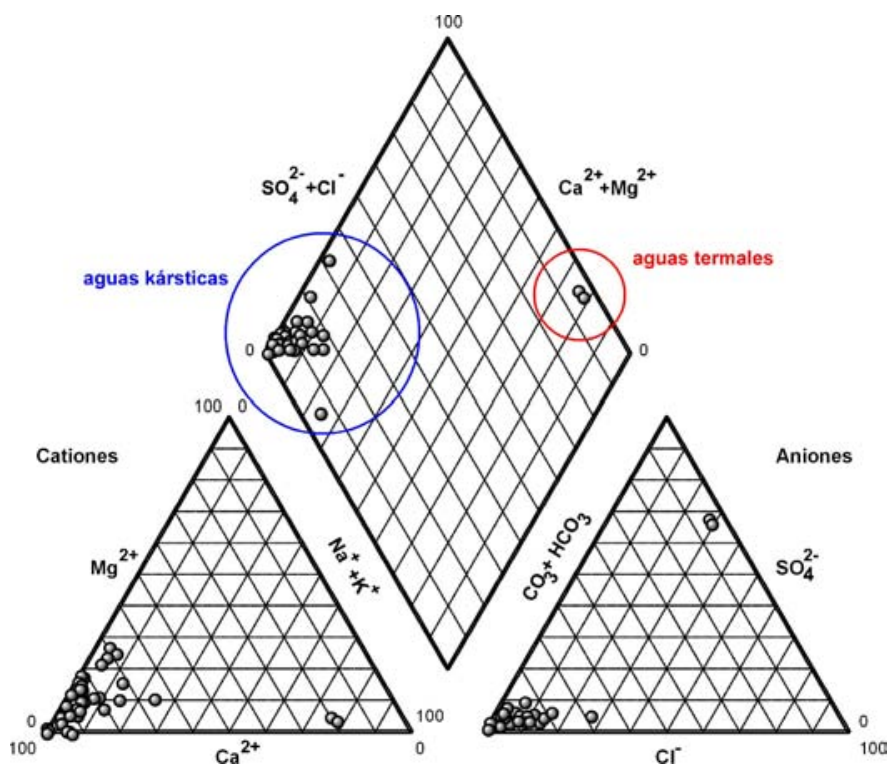

Figura 9. Diagrama Piper de 38 surgencias kársticas de los Picos de Europa (datos procedentes de la Base de Datos de Puntos de Agua del IGME - www.igme.es - y Ballesteros, 2016).

Figure 9. Piper Diagram of 38 karst springs of the Picos de Europa (data from the IGME Water Point Database - www.igme.es - and Ballesteros, 2016).

antaño, destacando su uso para el abastecimiento humano y la ganadería, actividad que junto al turismo, constituyen los principales motores económicos de la región (Rescia et al., 2008); localmente, el agua de los manantiales también se emplea en la generación de energía en pequeñas centrales hidroeléctricas y en mucha menor medida para regadío.

Las aguas subterráneas abastecen a más de 15 localidades del Parque Nacional y áreas limítrofes, cuya dotación total se estima en unos $15 \mathrm{hm} 3 /$ año. Esto puede suponer aproximadamente el $3 \%$ de los recursos totales disponibles. El agua subterránea también se emplea para dar servicio a la cabaña ganadera, cifrada para 2015 en más de 30.000 cabezas de ganado vacuno, ovino, caprino y equino (Llaneza y García, 2016), especialmente, en zonas situadas a una altitud superior a los $500 \mathrm{~m}$, donde vacas, ovejas y cabras pastan desde la primavera hasta el otoño.

Entre los usos del agua subterránea en el pasado, destaca su utilización hasta el siglo $\mathrm{XX}$, entre otras localidades, en Caín y Sajambre (León) y Tresviso (Cantabria), para dar servicio a molinos tradicionales, que desviaban parte del caudal de los manantiales. También se emplea para la generación de energía eléctrica, debido a la posición anómalamente alta de 
determinadas surgencias con respecto a los ríos principales. Así, en el Oriente de los Picos de Europa, las surgencias de El Nacimiento y Río Chico abastecen la central hidroeléctrica de Urdón, mientras que en la Garganta del Cares, las surgencias de Los Molinos proporcionan más del $50 \%$ del agua que recoge el canal que alimenta las centrales eléctricas de Poncebos y Arenas de Cabrales. Como reseña histórica, se podría mencionar que, para este fin, se intentó captar - sin éxito - las aguas de la surgencia El Farfáu de la Viña, situada $100 \mathrm{~m}$ por debajo del mencionado canal. En el Río Dobra, al Oeste de los Picos de Europa, la descarga del manantial del Reo Molín supone el 30-40 \% del agua embalsada por la presa de La Hocica, que alimenta la central de Restaño. En el pasado, las aguas subterráneas del acuífero kárstico también alimentaron la central hidroeléctrica de Las Pálvoras, situada en el $\mathrm{N}$ de los Picos de Europa. Se estima que, en total, se llegan a generar cerca de $100 \mathrm{MW}$ de potencia.

\section{Conclusiones}

Los estudios hidrogeológicos realizados fundamentalmente a partir de los años 90 s, constituyen una primera aproximación al funcionamiento del acuífero kárstico de los Picos de Europa, uno de los más importantes de la Cordillera Cantábrica desde el punto de vista medioambiental e hidrogeológico. Estos estudios incluyen inventarios de puntos de agua, ensayos de trazadores, modelos geométricos 3D del acuífero y análisis químicos, en combinación con una extensa documentación sobre cuevas kársticas elaborada por los equipos espeleológicos.

Esta investigación ha puesto de manifiesto la complejidad hidrogeológica del Parque Nacional de los Picos de Europa, como consecuencia de una estructura geológica compleja, condicionada por la presencia de numerosos cabalgamientos, fallas y plegamientos.

Destaca la existencia de un acuífero kárstico principal de edad Carbonífero Inferior (acuífero kárstico carbonífero), compartimentado debido a la presencia de acuitardos (acuitardo carbonífero detrítico en la Unidad Pisuerga-Carrión, acuitardo carbonífero sinorogénico, acuitardo de la secuencia del Carbonífero superior y acuitardo del Paleozoico Inferior en la Unidad Bodón-Ponga). La posición de los acuitardos condiciona, en gran parte, la existencia de variaciones en las cotas de los niveles piezométricos de los distintos compartimentos del acuífero y determinan que el sentido general del flujo tenga lugar hacia el $\mathrm{N}$. Sin embargo, localmente, el drenaje puede estar controlado por la estratificación y las fallas por lo que ocasionalmente toma otras direcciones. En líneas generales, el acuífero kárstico se comporta como libre, si bien, en determinadas ocasiones, como consecuencia de la posición de los acuitardos, puede comportarse como semiconfinado.

La velocidad de circulación estimada a partir de ensayos de trazador muestra valores dispares, con alta variabilidad, $y$, si bien se considera que en ocasiones se pueden superar los $300 \mathrm{~m} / \mathrm{h}$, los valores obtenidos son bajos con respecto a los publicados en otras áreas. Todo ello, hace que sea necesario recabar más datos para poder llegar a conclusiones fiables, siendo preciso establecer la continuidad en esta línea de investigación.

La recarga del acuífero kárstico se realiza principalmente por infiltración directa de las aguas de precipitación y fusión nival, a través del exokarst, así como a través de las formaciones superficiales cuaternarias (acuífero y acuitardo cuaternario). No obstante, de modo local, también se producen aportes procedentes de tramos de ríos influentes. El flujo en la zona vadosa del acuífero, está condicionado por el desarrollo del endokarst, siguiendo trayectorias subverticales a inclinadas y formando cursos de agua en las numerosas simas documentadas por los colectivos espeleológicos. La disposición E-O de los acuitardos y su inclinación generalizada al Norte, han condicionado la evolución de la karstificación y la orientación general del flujo de agua subterránea. La principal descarga del acuífero se realiza mediante grandes surgencias kársticas situadas a cotas entre 120 m s.n.m. (al $\mathrm{N}$ del límite del Parque) y $1300 \mathrm{~m}$ s.n.m. (Macizo Occidental). El caudal en algunas surgencias llega a exceder los $500 \mathrm{l} / \mathrm{s}$ en época de estiaje. Cabe destacar que en el Macizo Occidental la mayor compartimentación del acuífero lleva aparejado que la descarga de los distintos SGWBs tenga lugar a través de surgencias situadas a cotas que varían entre 200 y 1300 m s.n.m.

Las aguas subterráneas del Parque Nacional de los Picos de Europa son fundamentalmente bicarbonatadas cálcicas, generalmente subsaturadas en carbonatos, con presencia de cloruros procedentes de las nieblas asociadas al cercano Mar Cantábrico. También se puede destacar la presencia de aguas termales, asociadas a cabalgamientos, con temperaturas de hasta $60^{\circ} \mathrm{C}$, como las del Balneario de la Hermida, de naturaleza sulfatada.

El 3\% de las aguas de los Picos de Europa son empleadas para el abastecimiento humano y ganadero. Localmente, también se emplean como suministro de centrales hidroeléctricas, cuya potencia total instalada suma unos $100 \mathrm{MW}$.

Esta caracterización del acuífero kárstico establece 
una base de conocimiento sólida para la gestión de las aguas del Parque Nacional de los Picos de Europa, que debería considerar que el carácter compartimentado del acuífero kárstico, condiciona que los SGWBs presenten cotas de descarga y volúmenes diferentes, con distintas velocidades de flujo del agua subterránea y una dirección general del flujo hacia el $\mathrm{N}$, que es dónde se ubican los principales puntos de descarga. Por otra parte, sería recomendable desarrollar nuevos estudios relativos a la dinámica y quimismo de las aguas subterráneas para profundizar en el conocimiento hidrogeológico del Parque Nacional que, a día de hoy, sigue siendo escaso.

\section{Agradecimientos}

Agradecemos a las revisoras Beatriz Fernández González y Rosana Menéndez Duarte sus sugerencias de mejora del trabajo inicial; a Nemesio Heredia Carballo sus comentarios relativos a la geología de la zona; y a los diversos grupos espeleológicos, la información facilitada respecto a las cavidades subterráneas y ensayos de trazadores.

\section{Referencias}

Adrados, L.; Alonso, V.; Bahamonde, J.R.; Farias, P.; Fernández González, L.P.; Gutiérrez Claverol, M.; Heredia Carballo, N.; Jiménez Sánchez, M.; Meléndez Asensio, M.; Merino Tomé, O. y Villa Otero, E. (2010). Parque Nacional de los Picos de Europa. Guía Geológica. Guías Geológicas de Parques Nacionales. Adrados (ed.), 337 pp. (ISBN: 978-84-8014-786-6).

Alonso, J.L., Álvarez Díez, J., Peláez González, M., Rubio, A., 2009a. Evolución del conocimiento geológico y geotécnico en la zona de los Túneles de Pajares, in: Pando González, L., López Fernández, C., de la Rubia MIR, C. (eds.), Jornadas Técnicas de La Variante de Pajares. ADIFUniversidad de Oviedo, Oviedo, España, 61-75.

Alonso, J.L., Marcos, A., Suárez, A., 2009b. Paleogeographic inversion resulting from large out of sequence breaching thrusts:The León Fault (Cantabrian Zone, NW Iberia). A new picture of the external Variscan Thrust Belt in the Ibero-Armorican Arc. Geologica Acta 4, 451-473. doi:10.1344/105.000000

Alonso, J.L., Pulgar, J.A., García-Ramos, J.C., Barba, P., 1996. Tertiary basins and Alpine tectonics in the Cantabrian Mountains (NW Spain), in: Friend, P.F., Dabrio, C.J. (eds.), Tertiary Basins of Spain. Cambridge University Press, New York, 214-227.

Arquer Prendes-Pando, F., 2001. Los Picos de Europa, un depósito de agua constituido por la Naturaleza, in: Pozo Gómez, M. del, Durán-Valsero, J. (eds.), Agua Subterránea y Patrimonio Natural. Instituto Geológico y Minero de España, Madrid, pp. 304-327.
Austin, M., Wilcock, J., 1961. Water analysis and hydrology. Cave Res. Gr. Publ. 14, 11-20.

Bahamonde, J.R., Merino-Tomé, O., Heredia, N., 2007. A Pennsylvanian microbial boundstone-dominated carbonate shelf in a distal foreland margin (Picos de Europa Province, NW Spain). Sedimentary Geology, 198, 167-193. doi:10.1016/j.sedgeo.2006.12.004

Ballesteros, D., Jiménez-Sánchez, M., Giralt, S., DeFelipe, I., García-Sansegundo, J., 2017. Glacial origin for cave rhythmite during MIS $5 \mathrm{~d}-\mathrm{c}$ in a glaciokarst landscape, Picos de Europa (Spain). Geomorphology 286, 68-77. doi:10.1016/j.geomorph.2017.03.014

Ballesteros, D., 2016. Geomorfología y evolución geomorfológica de las cuevas alpinas de los Picos de Europa, Cordillera Cantábrica (España). Tesis Doctoral. Universidad de Oviedo, Oviedo. 325 p.

Ballesteros, D., Jiménez-Sánchez, M., Domínguez-Cuesta, M.J., García-Sansegundo, J., Meléndez-Asensio, M., 2015a. Geoheritage and Geodiversity Evaluation of Endokarst Landscapes: The Picos de Europa National Park, North Spain, in: Andreo, B., Carrasco, F., Durán, J.J., Jiménez, P., LaMoreaux, J.W. (eds.), Hydrogeological and Environmental Investigations in Karst Systems SE, Environmental Earth Sciences. Springer Berlin Heidelberg, Berlin, Germany, pp. 619-627. doi:10.1007/978-3-642-17435-3_69

Ballesteros, D., Malard, A., Jeannin, P.-Y., Jiménez-Sánchez, M., García-Sansegundo, J., Meléndez-Asensio, M., Sendra, G., 2015b. Influence of the rivers on speleogenesis combining KARSYS approach and cave levels. Picos de Europa, Spain, in: Andreo, B., Carrasco, F., Durán, J.J., Jiménez, P., LaMoreaux, J.W. (Eds.), Hydrogeological and Environmental Investigations in Karst Systems, Environmental Earth Sciences. Springer Berlin Heidelberg, Berlin, Germany, pp. 599-607. doi:10.1007/978-3-642-17435-3_67

Ballesteros, D., Malard, A., Jeannin, P.-Y., Jiménez-Sánchez, M., García-Sansegundo, J., Meléndez-Asensio, M., Sendra, G., 2015c. KARSYS hydrogeological 3D modeling of alpine karst aquifers developed in geologically complex areas. Picos de Europa National Park. Environ. Earth Science 74, 7699-7714. doi:10.1007/s12665-0154712-0

Ballesteros, D., Meléndez, M., Malard, A., Jiménez-Sánchez, M., Heredia, N., Jeannin, P.-Y., García-Sansegundo, J., 2014. Research in karst aquifers developed in highmountain areas combining KARSYS models with springs discharge records. Picos de Europa, Spain, in: EGU General Assembly, Viena, 014-497.

Ballesteros, D., Malard, A., Jeannin, P.-Y., Jiménez-Sánchez, M., García-Sansegundo, J., Meléndez, M., Sendra, G., 2013. Geometría y direcciones de flujo de aguas subterráneas preliminares de acuíferos kársticos mediante el método KARSYS. Picos de Europa, Norte de España, in: Alvarado-Berrezueta, E., Domínguez-Cuesta, M.J. (eds.), Técnicas Aplicadas a la Caracterización y Aprovechamiento de Recursos Geológico-Mineros. Red Minería XXI, CYTED, Instituto Geológico y Minero de España, 51-60.

Ballesteros, D., Puerta Elorza, E., Fernández Valencia, R. y 
Felipe Pitcairn, J. de. 2010. Torca Teyera. Subterránea, 30, 24-26

Barquín, J., Peñas, F., Álvarez-Cabria, M., Rodríguez-Castillo, T., González, A., Estévez, E., Lezcano, M., Fernández, F., Rojo, J., García, A., Álvarez, C., 2015. Optimización de las redes de seguimiento del estado de conservación en ríos de alta montaña, in: Amengual, P., Asensio, B. (eds.), Proyectos de Investigación en Parques Nacionales: 20102013. Organismo Autónomo de Parques Nacionales, Madrid, 35-61.

Borreguero, M., 1986. Special Picos: Puertos de Ondón. Informe inédito. Societé Speleogique de Suisse, Neuchâtel, Switzerland.

Carcavilla, L., Durán, J.J., García-Cortés, Á., López-Martínez, J., 2009. Geological Heritage and Geoconservation in Spain: Past, Present, and Future. Geoheritage 1, 75-91. doi:10.1007/s12371-009-0006-9

Collignon, B., 1986. Quelques elements de geologie et D’Hydrogeologie. Spelunca Supplément. 19, 7-12.

Deval Castillo, J., Luengo García, J. A., Lorenzo Riera, J. L., García Hernández J. and de Paz García, M. (2011). Módulo nival en los modelos hidrológicos de la Confederación Hidrográfica del Cantábrico. Calibración y validación en Picos de Europa. In: Jornada Internacional del Agua, Barcelona, España. ISBN-13: 978-84-615-4023-5.

Diaño Burlón y AD Cuasacas, 2011. 10/5/2017, http://gedburlon.blogspot.com.es

DGOH (1990). Unidades hidrogeológicas de España e Islas Baleares. Síntesis de sus características y mapa a escala 1:1.000.000. Servicio Geológico. Informaciones y Estudios $n^{\circ} 52$.

Durán Valsero, J., Robledo Ardila, P., 2009. Carbonate and evaporite karst systems of the Iberian Peninsula and the Baleatic Islands, in: García-Cortés, Á. (ed.), Spanish Geological Frameworks and Geosites. Instituto Geológico y Minero de España, Madrid, Spain, pp. 200-214.

Fabre, J. P. y Fabriol, R. 1984. Résultats de deux traçages réalisés dans l'amphithéâtre d'Ozania (Picos de Europa, Espagne). Karstologia, 4, 35-37.

Farias, P. y Heredia, N. (1994).- Geometría y Cinemática de los dúplex de Pambuches (Unidad de Picos de Europa, Zona Cantábrica, NO de España). Revista de la Sociedad Geológica de España, 7 (1-2), 113-120.

Fernández García, F., Rasilla, D. 1992. El viento en la Cornisa Cantábrica: avance de un estudio sobre los temporales del S. Cuadernos de Sección Historia, 20, 271-295.

Fernández-Martínez, E., Carcavilla, L., Heredia, N., Santos, J., Ballesteros, D., Fuertes, I., Adrados, L., 2017. Propuesta de inventario y geoindicadores en el Parque Nacional de los Picos de Europa. In: Patrimonio geológico, gestionando la parte abiótica del patrimonio natural. Cuadernos del Museo Geominero, Madrid, 21, 137 - 142.

Firman, H.A., 1979. The karst of the Eastern Massif, Picos de Europa. University of Lancaster, Lancaster.

Ford, D. C. 1993. Karst in cold environments. In: French, H. M. \& Slaymaker, O. (eds.) Canada's cold environments. McGill-Queen's University Press. Quebec. 199-222.

Gale, S. 1983. WaterTracing in the Western Picos de Europa,
Asturias, Northern Spain. Proceedings of the Oxford University Cave Club, 11, 33-35.

García Martin, 2018, L. Caracterización Hidrogeológica de Surgencias Termales en el Entorno de la Falla de Cabuérniga (Cantabria). Informe inédito (Trabajo de Fin de Master) Departamento de Geología, Universidad de Oviedo.

Garrido Ruiz, M., Serrano Martín, L., Míguez Bailo, R., Lombardero Barceló, M., Fernández-Serrano Sánchez, M., Álvarez Díez, J., 2009. El estudio hidrogeológico de los Túneles de Pájares. In: Pando González, L., López Fernández, C., de la Rubia MIR, C. (eds.), Jornadas Técnicas de La Variante de Pajares. ADIF-Universidad de Oviedo, Oviedo, España, 77-86

GE Diañu Burlón y AD Cuasacas. 2013. Canal de CanrasoLlanos del Burdio (Macizo Occidental-Picos de Europa). Informe Inédito. Exploraciones 2012, Corvera de Asturias.

Goldscheider, N., Meiman, J., Pronk, M. \& Smart, C. 2008. Tracer tests in karst hydrogeology and speleology. International Journal of Speleology, 37, 27-40.

Herguedas, B., Peña Puentes, E., del Olmo Ruiz, J., Álvarez Fernández, J.R., 2009 Modelo numérico de la relación túnel-aguas subterráneas. Galería de acceso del Lote 2 (Túneles de Pajares). In: Pando González, L., López Fernández, C., de la Rubia MIR, C. (eds.), Jornadas Técnicas de La Variante de Pajares. ADIF-Universidad de Oviedo, Oviedo, España, 87-93.

Herrero, N. 1992. Avance al estudio de las direcciones de drenaje del karst en el área sur de la peña Santa de Castilla (macizo occidental de los Picos de Europa, León). In: Bohigas, R. (ed.) Actas del $5^{\circ}$ Congreso Español de Espeleología. Federación Española de Espeleología. Camargo. 481-484.

Horsley, D., Roberts, S., Arthur, J. y Taylor, R. 1989. 1989 'Juracao' expedition final report. Oxford University Cave Club. Oxford.

IGME, 1982. Estudio hidrogeológico de la Cuenca Norte de España (Asturias). Fondo documental del Instituto Geológico y Minero de España: Código 36038. (15/9/2017, http://info.igme.es/ConsultaSID/presentacion.asp?ld=18829)

IGME, 1984. Investigación hidrogeológica de la Cuenca Norte: Asturias. Plan Nacional de Investigación de Aguas Subterráneas. Instituto Geológico y Minero de España, Madrid, Spain.

IGME, 2007 Mapa Litoestratigráfico, de permeabilidades e hidrogeológico de España a escala 1:200.000. Centro de documentación IGME, 17/6/2015, www.igme.es

IGME-DGA (2009). Actividad 4: Identificación y caracterización de la interrelación que se presenta entre aguas subterráneas, cursos fluviales, descargas por manantiales, zonas húmedas y otros ecosistemas naturales de especial interés hídrico. Demarcación Hidrográfica 016 Cantábrico. Masa de agua subterránea 016.214 Picos de Europa-Panes. Centro de documentación IGME, 25/1/2017, www.igme.es

Jędrzejczak, M. 2001. Western massif (El Cornion) of the Picos de Europa (Asturias, Spain). Publication of the 13th International Speleological Congress. Sociedade Brasileira de Espeleologia. Brasilia. 25-28. 
Jiménez-Sánchez, M., Rodríguez-Rodríguez, L., García-Ruiz, J.M., Domínguez-Cuesta, M.J., Farias, P., Valero-Garcés, B., Moreno, A., Rico, M., Valcárcel, M., 2013. A review of glacial geomorphology and chronology in northern Spain:Timing and regional variability during the last glacial cycle. Geomorphology 196, 50-64. doi:10.1016/j.geomorph.2012.06.009

Judson, D.M., 1963. Oxford-Derbyshire speleological expedition to NW Spain. Oxford University Cave Club, Oxford.

Karami, H. y Ashjari, J. 2010. Periodic breakthrough curve of tracer dye. Cave and karst science, 36, 5-10.

Liautaud, J.-P. 1985. 20 Ans de Spéléologie Aux Picos de Europa, Espagne (1964-1984). Montpellier. Spéléo Club Alpine Langedocien.

Llaneza, L. y García, E. J. 2016. Carga ganadera y daños a la ganadería. 30/11/2018, www.parquenacionalpicoseuropa.es

Lloyd, D.K., 1990. Water Tracing in the Vega Huerta Caves, Picos de Europa, Spain. Cave Science. 17, 103-106.

López Geta, J., Navarrete Martínez, P., Moreno Merino, L., Galindo Rodríguez, E., Meléndez Asensio, M., Pozo Gómez, M. del, 1997. In: Calidad química y contaminación de las aguas subterráneas en España, período 19821993. Cuenca del Norte. Instituto Tecnológico Geominero de España, Madrid, Spain.

López-Gómez, J., Fidel Martín González, F., Heredia Carballo, N., aúl de la Horra, R., Barrenechea, J.F., Cadenas, P., Juncal, M.; Díez, J. B., Borruel Abadía, V., Pedreira, D., García Sansegundo, J., Farias Arquer, P., Galé, C., Lago, M., Ubide, T., Fernández Viejo, G., Gand G. (2019). New lithostratigraphy for the Cantabrian Mountains: A common tectono-stratigraphic evolution for the onset of the Alpine cycle in the W Pyrenean realm, N Spain. Earth Science Reviews. 188, 249-271.

Lusarreta, J., 2007. Torca Castil. Plongeesout 3, 42-47.

MAGRAMA, 2014. Red de seguimiento del cambio global en Parques Nacionales, MAGRAMA, 16/9/2018, www.magrama.gob.es/es/red-parques-nacionales/redseguimiento/informes-meteorologicos.aspx

Marquínez, J., 1978. Estudio geológico del sector SE de los Picos de Europa (Cordillera Cantábrica, NW de España). Trabajos de Geología, 10, 295-315.

Marquínez, J. y Adrados, L., 2000. La geología y el relieve de los Picos de Europa. Naturalia Cantabricae 1: 3-19

Maurice, L. y Greaves, H. 2008. Dyetrace report. En: Graves, H. (ed.) Julagua 2008. A Caving and Cave Diving Expedition to the Picos de Europa, Asturias, Spain. Oxford University Cave Club. Oxford. 16-17.

Meléndez-Asensio, M., Nuño Ortega, C., Rebollar Quirós, A., 1998. Estudio hidrogeológico de Buferrera-Covadonga, Parque Nacional Picos de Europa (aplicación a la incidencia de un vertido de aguas residuales). Informe inédito, Centro de Documentación del Instituto Geológico y Minero de España, Oviedo.

Meléndez, M., Ballesteros, D., Jiménez-Sánchez, M., GarcíaSansegundo, J., 2015. Hydrogeological studies in high mountains karst environment: the example of Picos de Europa (Spain). Geophysical Research Abstracts, 17, EGU publications,13623-2.
Merino-Tomé, O., Bahamonde, J.R., Colmenero, J.R., Heredia, N., Villa, E., Farias, P., 2009. Emplacement of the Cuera and Picos de Europa imbricate system at the core of the Iberian-Armorican arc (Cantabrian zone, north Spain): New precisions concerning the timing of arc closure. Bulletin of the Geological Society of America. 121, 729-751. doi:10.1130/B26366.1

Merino-Tomé, O., Suárez Rodríguez, A., Alonso, J., 2013a. Mapa Geológico Digital continuo E. 1: 50.000, Zona Cantábrica (Zona-1000). GEODE. SIGECO-IGME. 22/6/2018, http://cuarzo.igme.es/sigeco/default.htm

Merino-Tomé, O., Suárez Rodríguez, A., Alonso, J., González Menéndez, L., Heredia, N., Marcos, A., 2013b. Mapa Geológico Digital continuo E. 1:50.000, Principado de Asturias (Zonas: 1100-1000-1600) [GEODE. SIGECOIGME. 22/6/2018, http://cuarzo.igme.es/sigeco/default.htm

Miotke, F.D. 1968. Karstmorphologische Studien in der glacial überformten höhenstufe der Picos de Europa, Nordspanien. Tesis Doctoral. Universität Hannover. Hannover. $161 \mathrm{p}$.

OAPN, 2016. Parque Nacional de los Picos de Europa. Seguimiento de datos socioeconómicos en las áreas de influencia socioeconómica. Informe de resultados para el periodo 2014-2016 MITECO, 25/2/2018, https://www. miteco.gob.es/es/red-parques-nacionales/nuestros-parques/picos-europa/2014-2016-picos_tcm30-439491.pdf

Ogando, E. 2007. Castil-Tortorios-Moñas 2006, un año más de exploraciones, un nuevo mil Piedras Verdes (-1.165 m). Subterránea, 27, 23-27.

Pullido, 2014. Principios de hidrogeología kárstica. Universidad Almería, $409 \mathrm{p}$.

Rescia, A., Pons, A., Lomba, I., Esteban, C., Dover, J., 2008. Reformulating the social-ecological system in a cultural rural mountain landscape in the Picos de Europa region (northern Spain). Landscape Urban Planning 88, 23-33.

Rodríguez Fernández, L.R., Heredia, N., 1987. La estratigrafía del carbonífero y la estructura de la Unidad del Pisuerga- Carrión. Cuadernos do Laboratorio Xeolóxico de Laxe 12, 207-229.

Ruiz-Fernández, J., Oliva, M., Cruces, A., Lopes, V., Freitas, M. da C., Andrade, C., García-Hernández, C., López-Sáez, J.A., Geraldes, M., 2016. Environmental evolution in the Picos de Europa (Cantabrian Mountains, SW Europe) since the Last Glaciation. Quaternary Science Reviews, 138, 87-104. doi:10.1016/j.quascirev.2016.03.002

Ruiz-Fernández, J., Poblete Piedrabuena, M., 2012. Las simas del macizo Occidental de los Picos de Europa: disposición estructural, depósitos asociados y características del drenaje. Investigaciones Geográficas 57, 205-223.

Serrano, E., González-Trueba, J.J., Pellitero, R., GonzálezGarcía, M., Gómez-Lende, M., 2013. Quaternary glacial evolution in the Central Cantabrian Mountains (Northern Spain). Geomorphology 196, 65-82. doi:10.1016/j.geomorph.2012.05.001

Smart, P. The geology, geomorphology and speleogenesis of the Eastern Massifs, Picos de Europa, North Spain. Cave Science, 11-4.

Struckmeier and Margat, (1995) Guide and Standard Legend 
for hydrogeological maps, Volume 17, International Association of Hydrogeologists, Hannover

Valenzuela, P., Domínguez-Cuesta, M.J., Meléndez-Asensio, M., Jiménez-Sánchez, M., de Santa María, J.A.S., 2015. Active sinkholes: A geomorphological impact of the Pajares Tunnels (Cantabrian Range, NW Spain). Engineering Geology 196, 158-170. doi:10.1016/j.enggeo.2015.07.007

Virgós Soriano, L., 1981. Estudios hidrogeológicos de los Picos de Europa. Aprovechamiento energético de los acuíferos. Informe Inédito. Centro de documentación del IGME, Oviedo, Spain.

Virgós Soriano, L., Pendás Fernández, F., Arquer PrendesPando, F., 1980. Sistemas hidrogeológicos de los Picos de Europa. Consideraciones sobre un eventual aprovechamiento energético de los acuíferos. VI Jornadas Nacionales Minerometalúrgicas. Asociación Nacional de Ingenieros de Minas, Huelva, Spain, 19-137.

Willis, R. 1981. Pozu del Xitu - 1981 dye tests. Proceeding of the Oxford University Cave Club, 10, 49-50.

Recibido: julio 2018

Revisado: septiembre 2018

Aceptado: febrero 2019

Publicado: diciembre 2019 